\title{
Coulomb screening and electronic instabilities of small-diameter $(5,0)$ nanotubes
}

\author{
J. González ${ }^{1}$ and E. Perfetto ${ }^{1,2}$ \\ ${ }^{1}$ Instituto de Estructura de la Materia, Consejo Superior de Investigaciones Científicas, Serrano 123, 28006 Madrid, Spain \\ ${ }^{2}$ Istituto Nazionale di Fisica Nucleare-Laboratori Nazionali di Frascati, Via E. Fermi 40, 00044 Frascati, Italy
}

(Received 13 July 2005; published 4 November 2005)

\begin{abstract}
We investigate the instabilities that may lead to the breakdown of the Luttinger liquid in the small-diameter $(5,0)$ nanotubes, paying attention to the competition between the effective interaction mediated by phonon exchange and the Coulomb interaction. We use bosonization methods to achieve an exact treatment of the Coulomb interaction at small momentum transfer, and apply next renormalization group methods to analyze the low-energy behavior of the electron system. This allows us to discern the growth of several response functions for charge-density-wave modulations and for superconducting instabilities with different order parameters. We show that, in the case of single nanotubes exposed to screening by external gates, the Luttinger liquid is unstable against the onset of a strong-coupling phase with very large charge-density-wave correlations. The temperature of crossover to the new phase depends crucially on the dielectric constant $\kappa$ of the environment, ranging from $T_{c} \sim 10^{-4} \mathrm{~K}$ (at $\kappa \approx 1$ ) up to a value $T_{c} \sim 10^{2} \mathrm{~K}$ (reached from $\kappa \approx 10$ ). The physical picture is however different when we consider the case of a large array of nanotubes, in which there is a three-dimensional screening of the Coulomb interaction over distances much larger than the intertube separation. The electronic instability is then triggered by the divergence of one of the charge stiffnesses in the Luttinger liquid, implying a divergent compressibility and the appearance of a regime of phase separation into spatial regions with excess and defect of electron density.
\end{abstract}

DOI: 10.1103/PhysRevB.72.205406

\section{INTRODUCTION}

During the last decade, carbon nanotubes have shown a great potential for the development of molecular electronics. This comes from their versatile electronic properties, which depend on variables like the helicity of the tubule, the temperature, or the contacts used in the experiments. It is wellknown for instance that the carbon nanotubes may have semiconducting or metallic behavior, depending on the roll-up direction of the wrapped hexagonal carbon lattice. ${ }^{1}$ This has opened the way for the construction of intramolecular junctions behaving as electronic devices at the nanometer scale. $^{2}$

Given their reduced dimensionality, the metallic nanotubes behave as strongly correlated electron systems, with different regimes depending on the energy scale. At room temperature, for instance, the strong Coulomb repulsion prevails in nanotubes of typical radius, driving the electron system to a state with the properties of the so-called Luttinger liquid, characterized by the absence of electron quasiparticles at the Fermi level. This feature manifests in the power-law dependence of observables such as the tunneling density of states, whose suppression at low energies has been actually observed in measurements of the conductance in the carbon nanotubes. ${ }^{2,3}$

At low temperatures, the behavior of the carbon nanotubes depends on the contacts used in the transport measurements. When the contacts are not highly transparent, there is in general a suppression of the zero-bias conductance and the differential conductivitiy, characteristic of the Coulomb blockade regime. On the other hand, there have been experiments using high-quality contacts where it has been possible to observe superconducting correlations in the carbon nanotubes. ${ }^{4,5}$ The most remarkable signature has been the appearance of supercurrents in nanotube ropes suspended be-
PACS number(s): 73.63.Fg, 71.10.Pm, 74.20.Mn

tween superconducting electrodes. ${ }^{4}$ This feature may be understood as a consequence of the proximity effect, by which Cooper pairs are formed in the nanotubes near the superconducting electrodes. ${ }^{6}$ Moreover, superconducting transitions have been measured in ropes suspended between metallic, nonsuperconducting electrodes. ${ }^{7,8}$ These observations imply the existence of a regime with a relevant attractive component in the electron-electron interaction, coming presumably from the exchange of phonons between electronic currents. ${ }^{9}$

From a theoretical point of view, it is well-known that the Luttinger liquid regime may break down in the carbon nanotubes, due to a variety of low-temperature instabilities. ${ }^{10-15}$ When the effective phonon-mediated interactions are taken into account, it can be shown that the superconducting correlations may grow large upon suitable screening of the Coulomb interaction in the nanotubes. ${ }^{16,17}$ This has raised the hopes that such correlations could be amplified by considering nanotube geometries with enhanced electron-phonon couplings. The case of the small-diameter nanotubes, with radius down to $0.2 \mathrm{~nm}$, is particularly interesting in that respect, since the large curvature of the tubule increases significantly the coupling to the elastic modes of the nanotube lattice. ${ }^{18,19}$

The transport properties of small-diameter nanotubes have been studied in the experiment reported in Ref. 20, where it has been claimed as evidence for a superconducting transition at about $15 \mathrm{~K}$. It is uncertain however whether the observed features should be ascribed to the presence of nanotubes with the $(5,0)$ or the $(3,3)$ geometry, both being consistent with the measured nanotube radius. Moreover, the physical meaning of the estimated transition temperature also remains unclear, as the nanotubes in the experiment are placed in the channels of a zeolite matrix that does not allow coherent electron hopping between the nanotubes. 


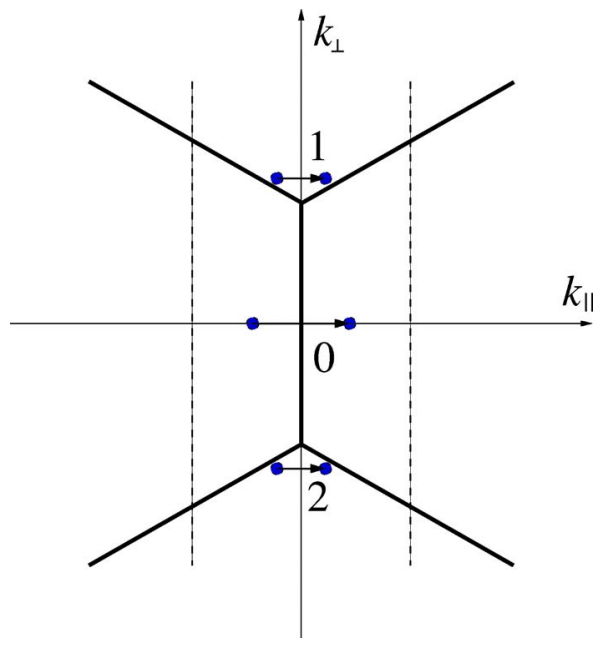

FIG. 1. (Color online) Representation of the Brillouin zone of the $(5,0)$ nanotubes (region between vertical dashed lines) showing the position of the Fermi points belonging to the low-energy degenerate subbands (denoted by 1,2) and to the zero angular-momentum subband (denoted by 0 ). The thick segments represent the boundaries of the hexagonal Brillouin zone for the graphene sheet.

The aim of the present paper is to investigate the instabilites that may lead to the breakdown of the Luttinger liquid in the small-diameter zigzag nanotubes. We are going to focus on the analysis of the $(5,0)$ nanotubes, which deserve special attention as they have three subbands crossing the Fermi level (in the undoped system). ${ }^{21,22}$ The position of the different Fermi points in momentum space is shown in Fig. 1. They correspond to two degenerate subbands with opposite values of the angular momentum around the nanotube axis (denoted by 1,2) and a third subband with zero angular momentum (labeled by 0 in the figure). The Fermi velocity is different in the nondegenerate and the degenerate subbands, being smaller in either case than for nanotubes of normal radius and leading to an enhanced density of states at low energies. For the degenerate subbands, the Fermi velocity becomes $v_{F} \approx 2.8 \times 10^{5} \mathrm{~ms}^{-1}$, while for the nondegenerate subband we have $v_{F}^{(0)} \approx 6.9 \times 10^{5} \mathrm{~ms}^{-1}$. A diagram with the different shapes of the subbands along the momentum in the longitudinal direction is shown in Fig. 2.

Together with the enhanced density of states at low energies, the large curvature of the tubule makes the $(5,0)$ geometry the most appealing instance to study the effects of large electronic correlations in the carbon nanotubes. We will apply renormalization group methods ${ }^{23}$ to discern the lowenergy instabilities of the electron system with three subbands at the Fermi level, extending the analysis carried out in Refs. 11 and 24 for typical nanotubes with two low-energy subbands. This will allow us to study the growth of several response functions marking the tendency towards long-range order, including charge-density-wave (CDW) modulations with different values of the momentum and superconducting instabilities with different order parameters.

Our analysis will stress the relevance that the screening of the Coulomb interaction has for the development of different instabilities in the small-diameter nanotubes. For a single nanotube, placed in general above some substrate, there is

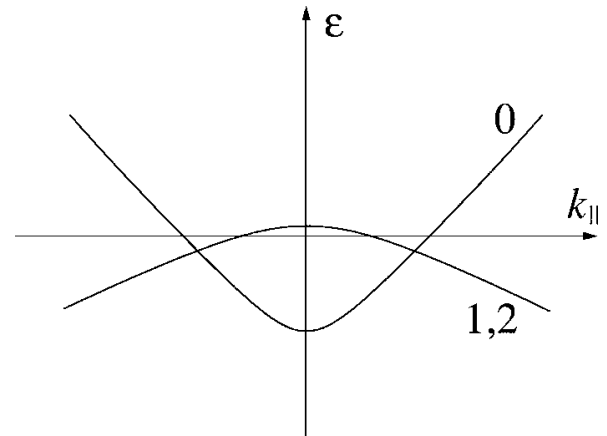

FIG. 2. Schematic representation of the dispersion of the lowenergy degenerate subbands (superposed in the curve labeled by 1 and 2) and the zero angular-momentum subband (labeled by 0 ) of the $(5,0)$ nanotubes. The Fermi level corresponds to $\varepsilon=0$ (in the undoped system).

little reduction of the Coulomb potential, which remains long-ranged in the one-dimensional (1D) system. ${ }^{25}$ The Coulomb interaction has then a strong effective coupling, much larger than that of the effective phonon-mediated interaction, so that the Coulomb repulsion plays the dominant role dictating the electronic properties about room temperatures. We will use bosonization methods ${ }^{26}$ to achieve an exact treatment of the contributions from the Coulomb interaction at small momentum transfer, capturing the effects of the strong Coulomb repulsion at the nonperturbative level. The contributions from the effective phonon-exchange interactions will be analyzed next, assessing their enhancement at low energies from the nontrivial scaling of the backscattering interaction channels.

On the other hand, we will also pay attention to the particular conditions in the experiment reported in Ref. 20. Each nanotube is embedded there in a large three-dimensional (3D) array of nanotubes. Thus, the electrostatic coupling between charges in the different nanotubes leads to a large screening of the Coulomb potential. We will study this effect by using a kind of generalized random-phase approximation (RPA) scheme, taking into account the Coulomb interaction between all the nanotubes in the 3D array. We will show that, over distances much larger than the intertube separation, there is a regime where the nanotube array screens effectively as a $3 \mathrm{D}$ system, rendering short-ranged the Coulomb potential within each nanotube. This is the most important effect of the environment in the particular experimental setup described in Ref. 20. We will see that the strong reduction of the Coulomb interaction leads then to the prevalence of charge instabilities with similar character to the WentzelBardeen singularity. ${ }^{27}$

We may compare the results of our investigation with those obtained for the small-diameter zigzag nanotubes by means of $a b$ initio simulations ${ }^{28}$ and mean-fieldlike calculations. ${ }^{29}$ In Ref. 29, a superconducting instability has been found to be dominant in the $(5,0)$ nanotubes, at a scale of about $1 \mathrm{~K}$. On the other hand, a CDW instability has been identified in the same geometry at room temperature in Ref. 28 (see also Ref. 30 for the Peierls instability in armchair nanotubes). Our results stress that the scale and the type of electronic instability are very sensitive to the degree of 
screening applied to the nanotubes. We will find indeed that single $(5,0)$ nanotubes (with just some screening from nearby gates) undergo the breakdown of the Luttinger liquid regime in favor of a CDW modulation at low energies. This is quite different from the instability found in the 3D array of nanotubes. In either case, we will see that the onset of the electronic instability takes place at temperatures ranging from $T_{c} \sim 10^{-4} \mathrm{~K}$ up to $\sim 10^{2} \mathrm{~K}$, depending on the dielectric constant of the environment.

\section{LUTTINGER LIQUID APPROACH TO ELECTRONIC PROPERTIES}

As a first approximation to the electronic properties of the small-diameter nanotubes, we begin our analysis by focusing on the effects of the strong Coulomb repulsion. This is the dominant interaction in scattering processes at low momentum transfer. Following Ref. 11, we take the Coulomb potential in the wrapped geometry as

$$
V_{C}\left(\mathbf{r}-\mathbf{r}^{\prime}\right)=\frac{e^{2} / \kappa}{\sqrt{\left(x-x^{\prime}\right)^{2}+4 R^{2} \sin ^{2}\left[\left(y-y^{\prime}\right)^{2} / 2 R^{2}\right]+a_{z}^{2}}},
$$

where $a_{z} \simeq 1.6 \AA$ and $R$ is the nanotube radius. The effective strength of the interaction depends on the dielectric properties of the environment hosting the nanotubes. In general, we will encode the screening effects from external gates in the dielectric constant $\kappa$. We have to bear in mind, however, that this procedure is suitable for the description of single nanotubes, while it has to be appropriately improved for systems made of a manifold of nanotubes, as we will see in Sec. IV.

The Fourier transform $\tilde{V}_{C}(k, q)$ of the potential (1) reflects the long-range character of the interaction at small momentum transfer. Thus, it has a logarithmic dependence at small longitudinal momentum $k$, which is characteristic of the 1D Coulomb potential in momentum space ${ }^{31}$

$$
\tilde{V}_{C}(k, 0) \approx \frac{2 e^{2}}{\kappa} \ln \left(\frac{k_{c}+k}{k}\right),
$$

where $k_{c}$ is in general of the order of the inverse of the nanotube radius $R$, as it is the memory that the electron system keeps of the finite transverse size, after projection of the 3D potential onto the longitudinal direction of the tubule. The strength of the Coulomb interaction can be estimated from the dimensionless ratio $e^{2} / \kappa v_{F}$, which for the smalldiameter nanotubes is of the order of $\sim 8 / \kappa$. This gives a measure of the relevance of the Coulomb interaction, which turns out to be in the strong-coupling regime in the forwardscattering channels.

Before going ahead, we proceed next to make a complete catalogue of the different interaction channels. We first borrow the classification that has been made already for metallic nanotubes with a pair of subbands at the Fermi level. ${ }^{24}$ Thus, we introduce respective coupling constants $g_{i}^{(j)}$ for the channels involving processes between the two inner subbands of the small-diameter nanotubes. The lower index discerns whether the interacting particles shift from one subband to
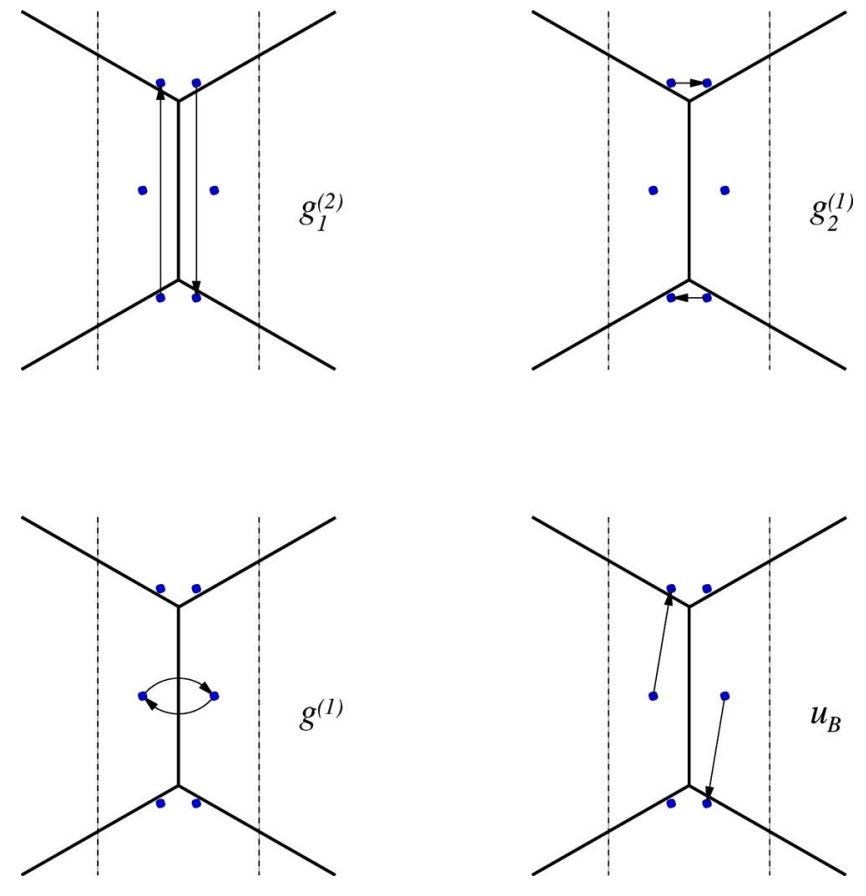

FIG. 3. (Color online) Diagrams showing the shift in momentum space of the interacting electrons and the momentum transfer involved in different backscattering processes in the $(5,0)$ nanotubes.

the other $(i=1)$, remain at different subbands $(i=2)$, or interact within the same subband $(i=4)$. The upper label follows the same rule to classify the different combinations of left movers and right movers.

The above set of couplings $g_{i}^{(j)}$ has to be supplemented with the couplings for the channels involving processes between a particle at one of the degenerate subbands and another at the nondegenerate subband. Now, we can discern between interactions that keep each particle within its respective subband, to which we assign a coupling $f^{(2)}\left(f^{(4)}\right)$ for particles of opposite (like) chirality, and backscattering interactions that lead to the exchange of the subbands for the two particles, which we label with a coupling $f^{(1)}$. Moreover, we have also the channels in which the particles interact within the outer subband, and that we will label as in the usual $g$-ology description, in terms of the couplings $g^{(1)}, g^{(2)}$, and $g^{(4)}$. Finally, there are also interaction processes in which two particles with about zero total momentum exchange their position from the outer to the inner subbands, or vice versa, as depicted in Fig. 3. We will assign the coupling $u_{F}$ to that kind of interaction with no change of chirality of the particles, and the coupling $u_{B}$ when there is a change in their chirality. It is worthwhile to remark that, despite the role they may have in the enhancement of some type of correlations, these interactions labeled by $u_{F}$ and $u_{B}$ have been overlooked in previous studies of the $(5,0)$ nanotubes. ${ }^{32}$

Focusing again on the forward-scattering interactions, we may represent their contribution to the Hamiltonian of the electron system introducing the charge and spin density operators

$$
\rho_{r i}(x)=\frac{1}{\sqrt{2}}\left[\Psi_{r i \uparrow}^{\dagger}(x) \Psi_{r i \uparrow}(x)+\Psi_{r i \downarrow}^{\dagger}(x) \Psi_{r i \downarrow}(x)\right],
$$




$$
\sigma_{r i}(x)=\frac{1}{\sqrt{2}}\left[\Psi_{r i \uparrow}^{\dagger}(x) \Psi_{r i \uparrow}(x)-\Psi_{r i \downarrow}^{\dagger}(x) \Psi_{r i \downarrow}(x)\right],
$$

which correspond to the different electron fields $\Psi_{\text {ri }}$ for the linear branches about the Fermi points shown in Fig. 2. We adopt a notation in which the index $r=L, R$ is used to label the left- or right-moving character of the linear branch, and the index $i=0,1,2$ to label the subband. We will assume that the interaction mediated by phonon exchange (as well as the Coulomb interaction) does not depend on the spin projection of the interacting electrons. This will allow us to take equal couplings for the interactions between electrons with the same and the opposite spin projection, leading to a description just in terms of the charge density operators.
For the sake of organizing the different forward-scattering interactions, it is convenient to define the symmetric and antisymmetric combinations of corresponding density operators in the two degenerate subbands

$$
\begin{aligned}
& \rho_{R \pm}(k)=\frac{1}{\sqrt{2}}\left[\rho_{R 1}(k) \pm \rho_{R 2}(k)\right], \\
& \rho_{L \pm}(k)=\frac{1}{\sqrt{2}}\left[\rho_{L 2}(k) \pm \rho_{L 1}(k)\right] .
\end{aligned}
$$

With this change of variables, the Hamiltonian for the forward-scattering interactions can be written in the form

$$
\begin{aligned}
H_{F S}= & \frac{1}{2} v_{F} \int_{-k_{c}}^{k_{c}} d k \sum_{r=L, R} \sum_{i= \pm} \rho_{r i}(k) \rho_{r i}(-k),+\frac{1}{2} v_{F}^{(0)} \int_{-k_{c}}^{k_{c}} d k \sum_{r=L, R} \rho_{r 0}(k) \rho_{r 0}(-k)+\frac{1}{2} \int_{-k_{c}}^{k_{c}} \frac{d k}{2 \pi} 2\left(\rho_{R+}(k)\left(g_{4}^{(4)}+g_{2}^{(4)}\right) \rho_{R+}(-k)+\rho_{L+}(k)\left(g_{4}^{(4)}\right.\right. \\
& \left.+g_{2}^{(4)}\right) \rho_{L+}(-k)+\rho_{R-}(k)\left(g_{4}^{(4)}-g_{2}^{(4)}\right) \rho_{R-}(-k)+\rho_{L-}(k)\left(g_{4}^{(4)}-g_{2}^{(4)}\right) \rho_{L-}(-k)+2 \rho_{R+}(k)\left(g_{4}^{(2)}+g_{2}^{(2)}\right) \rho_{L+}(-k)+2 \rho_{R-}(k)\left(g_{2}^{(2)}\right. \\
& \left.\left.-g_{4}^{(2)}\right) \rho_{L-}(-k)\right)+\frac{1}{2} \int_{-k_{c}}^{k_{c}} \frac{d k}{2 \pi} 2\left(\rho_{R 0}(k) g^{(4)} \rho_{R 0}(-k)+\rho_{L 0}(k) g^{(4)} \rho_{L 0}(-k)+2 \rho_{R 0}(k) g^{(2)} \rho_{L 0}(-k)\right) \\
& +\frac{1}{2} \int_{-k_{c}}^{k_{c}} \frac{d k}{2 \pi} 4 \sqrt{2}\left(\rho_{R+}(k) f^{(4)} \rho_{R 0}(-k)+\rho_{L+}(k) f^{(4)} \rho_{L 0}(-k)+\rho_{R+}(k) f^{(2)} \rho_{L 0}(-k)+\rho_{L+}(k) f^{(2)} \rho_{R 0}(-k)\right)
\end{aligned}
$$

where $k_{c}$ stands again for the momentum cutoff dictated by the transverse size of the nanotube.

In order to diagonalize the Hamiltonian (7), we introduce the fields $\Phi_{+}(x), \Phi_{-}(x), \Phi_{0}(x)$ [and their respective conjugate momenta, $\left.\Pi_{+}(x), \Pi_{-}(x), \Pi_{0}(x)\right]$ having the following relation with the electron density operators:

$$
\begin{aligned}
& \partial_{x} \Phi_{+}(x)=\sqrt{\pi}\left[\rho_{L+}(x)+\rho_{R+}(x)\right], \\
& \partial_{x} \Phi_{-}(x)=\sqrt{\pi}\left[\rho_{L-}(x)+\rho_{R-}(x)\right], \\
& \partial_{x} \Phi_{0}(x)=\sqrt{\pi}\left[\rho_{L 0}(x)+\rho_{R 0}(x)\right] .
\end{aligned}
$$

In terms of these fields, we can write the Hamiltonian (7) in the form

$$
\begin{aligned}
H_{F S}= & \frac{1}{2} u_{+} \int d x\left(K_{+}\left[\Pi_{+}(x)\right]^{2}+\left(1 / K_{+}\right)\left[\partial_{x} \Phi_{+}(x)\right]^{2}\right) \\
& +\frac{1}{2} u_{-} \int d x\left(K_{-}\left[\Pi_{-}(x)\right]^{2}+\left(1 / K_{-}\right)\left[\partial_{x} \Phi_{-}(x)\right]^{2}\right) \\
& +\frac{1}{2} u_{0} \int d x\left(K_{0}\left[\Pi_{0}(x)\right]^{2}+\left(1 / K_{0}\right)\left[\partial_{x} \Phi_{0}(x)\right]^{2}\right) \\
& +\frac{1}{2} \int d x \frac{2 \sqrt{2}}{\pi}\left(\Pi_{+}(x)\left(f^{(4)}-f^{(2)}\right) \Pi_{0}(x)+\partial_{x} \Phi_{+}(x)\right. \\
& \left.\times\left(f^{(4)}+f^{(2)}\right) \partial_{x} \Phi_{0}(x)\right) .
\end{aligned}
$$

The renormalized velocities $u_{+}, u_{-}, u_{0}$ and charge stiffnesses $K_{+}, K_{-}, K_{0}$ are given by the equations

$$
\begin{gathered}
u_{ \pm} K_{ \pm}=v_{F}+(1 / \pi)\left(g_{4}^{(4)} \pm g_{2}^{(4)}-\left(g_{2}^{(2)} \pm g_{4}^{(2)}\right)\right), \\
u_{ \pm} / K_{ \pm}=v_{F}+(1 / \pi)\left(g_{4}^{(4)} \pm g_{2}^{(4)}+\left(g_{2}^{(2)} \pm g_{4}^{(2)}\right)\right), \\
u_{0} K_{0}=v_{F}^{(0)}+(1 / \pi)\left(g^{(4)}-g^{(2)}\right), \\
u_{0} / K_{0}=v_{F}^{(0)}+(1 / \pi)\left(g^{(4)}+g^{(2)}\right) .
\end{gathered}
$$

At this stage, the Hamiltonian (11) can be brought to diagonal form by (i) applying a simple canonical transformation

$$
\begin{aligned}
& \Phi_{+}=\sqrt{K_{+}} \widetilde{\Phi}_{+}, \quad \Pi_{+}=\frac{1}{\sqrt{K_{+}}} \widetilde{\Pi}_{+}, \\
& \Phi_{-}=\sqrt{K_{-}} \widetilde{\Phi}_{-}, \quad \Pi_{-}=\frac{1}{\sqrt{K_{-}}} \widetilde{\Pi}_{-}, \\
& \Phi_{0}=\sqrt{K_{0}} \widetilde{\Phi}_{0}, \quad \Pi_{0}=\frac{1}{\sqrt{K_{0}}} \widetilde{\Pi}_{0},
\end{aligned}
$$

and (ii) making an additional rotation to disentangle the $(+, 0)$ sector 


$$
\begin{gathered}
\tilde{\Phi}_{0}=c_{0} \sqrt{\mu} \hat{\Phi}_{0}+s_{0} \sqrt{\nu} \hat{\Phi}_{+}, \\
\tilde{\Pi}_{0}=\frac{c_{0}}{\sqrt{\mu}} \hat{\Pi}_{0}+\frac{s_{0}}{\sqrt{\nu}} \hat{\Pi}_{+}, \\
\tilde{\Phi}_{+}=c_{+} \sqrt{\beta} \hat{\Phi}_{+}+s_{+} \sqrt{\alpha} \hat{\Phi}_{0}, \\
\widetilde{\Pi}_{+}=\frac{c_{+}}{\sqrt{\beta}} \hat{\Pi}_{+}+\frac{s_{+}}{\sqrt{\alpha}} \hat{\Pi}_{0},
\end{gathered}
$$

where the parameters $c_{0}, s_{0}, c_{+}, s_{+}, \alpha, \beta, \mu, \nu$ are evaluated in Appendix A. The important point is that, at the end, the excitations of the system governed by the Hamiltonian (11) are given by charge fluctuations, with velocities strongly renormalized by the interactions, and which constitute the whole spectrum together with the spin fluctuations propagating with the unrenormalized velocities $v_{F}$ and $v_{F}^{(0)}$.

The picture that we have just developed corresponds to the Luttinger liquid regime of the electron system. This regime holds at energies where there is approximate linear dispersion of the different subbands crossing the Fermi level, which places typically an upper scale $E_{c}$ of the order of $\sim 0.1 \mathrm{eV}$. In the case of single nanotubes, the dominant contribution to the forward-scattering couplings in (7) comes from the strong Coulomb potential in (2), with some infrared cutoff which is dictated in general by the longitudinal size of the nanotube. ${ }^{11}$ This leads to values of the charge stiffnesses that are typically smaller than 1 and in agreement with the experimental observations of Luttinger liquid behavior in the carbon nanotubes. ${ }^{2,3}$ For energy scales much lower than $E_{c}$, however, the rest of the interaction channels neglected in the above analysis may become as important as the forwardscattering channels. This is possible as the strength of the 1D interactions depends in general on the energy scale of the interaction processes. ${ }^{23}$ Electronic instabilities are then expected when some of the backscattering interactions leave the weak-coupling regime as the temperature or other relevant energy scale is lowered, as we analyze next in the case of the small-diameter $(5,0)$ nanotubes.

\section{BACKSCATTERING INTERACTIONS AND LOW- ENERGY INSTABILITIES}

In general, the exchange of phonons between electronic currents gives rise to a retarded interaction, which can be represented by the effective potential obtained by integrating out the phonons in the many-body theory. ${ }^{27}$ If we denote the electron-phonon couplings by $g_{i i^{\prime}}(k)$ (labeling by $i, i^{\prime}$ the subbands of the incoming and the outgoing electron), we can express the potential for the effective electron-electron interaction in the form

$$
V_{i j, i^{\prime} j^{\prime}}(k, \omega)=-2 g_{i i^{\prime}}(k) g_{j j^{\prime}}(-k) \frac{\omega_{k}}{-\omega^{2}+\omega_{k}^{2}},
$$

where $\omega_{k}$ stands for the energy of the exchanged phonon with momentum $k$. The typical energy of the phonons in the optical branches (or in the acoustic branches at large momen- tum transfer) is of the order of $\sim 0.1 \mathrm{eV},{ }^{29}$ and therefore comparable to the energy cutoff $E_{c}$ of the $1 \mathrm{D}$ electron system. This allows us to take the interaction arising from (18) as a source of attraction, in the energy range where the 1D model makes sense.

The electron-phonon couplings in the $(5,0)$ nanotubes have been analyzed with great detail in Ref. 29. It has been shown there that the couplings with greater strength correspond to intraband processes, for which the momentum transfer is in the longitudinal direction. Following for instance the results of that reference for the backscattering processes within subband 1 (or subband 2), we find that the strength of the effective electron-electron interaction with $2 k_{F}$ momentum-transfer is given by the coupling

$$
\sum_{\nu} 2\left(g_{11}^{(\nu)}\left(2 k_{F}\right)\right)^{2} / v_{F} \omega_{2 k_{F}}^{(\nu)} \approx 0.6
$$

after summing over the different phonon modes with nonvanishing electron-phonon coupling. ${ }^{29}$ Similarly, for backscattering processes within the nondegenerate subband, we find the effective coupling

$$
\sum_{\nu} 2\left(g_{00}^{(\nu)}\left(2 k_{F}^{(0)}\right)\right)^{2} / v_{F} \omega_{2 k_{F}^{(\nu)}}^{(\nu)} \approx 1.1
$$

The first of these values represents the contribution of the attractive phonon-mediated interaction to the couplings $g_{2}^{(1)}$ and $g_{4}^{(1)}$, while the second value provides the contribution to the coupling $g^{(1)}$. Making similar estimates of the electronphonon couplings and frequencies at large transverse momentum transfer, we have found that the couplings for the rest of the backscattering interactions $g_{1}^{(1)}, g_{1}^{(2)}, f^{(1)}, u_{F}$, and $u_{B}$ are given by $\approx 0.5 v_{F}$.

For each of these backscattering contributions, one has to subtract an opposite correction from the Coulomb interaction. This is given by the Fourier transform $\tilde{V}_{C}(k, q)$ of the potential in (1) at the corresponding momentum transfer, with an additional factor of reduction that comes from the particular structure of the Bloch functions in the smalldiameter nanotubes. ${ }^{29}$ In general, the larger contributions from the Coulomb potential are found in the channels with the smaller momentum transfer. The forward-scattering channels deserve special consideration, as the potential (2) has to be evaluated with a cutoff at small $k$. As already mentioned, this is provided in general by the length $L$ of the experimental samples, so that a sensible estimate is given typically by a spatial average of the potential $\tilde{V}_{C}(k \approx 0,0)$ $\approx\left(2 e^{2} / \kappa\right) \ln \left(k_{c} / k_{0}\right)$, with $k_{0} \sim 1 / L \sim 10^{-3} k_{c}$.

In this section we analyze the instabilities of single nanotubes, so that we can disregard for the moment any kind of intertube interaction. Then, taking into account the contributions from the phonon exchange and the Coulomb interaction, we obtain the following values for the couplings in the different interaction channels:

$$
\begin{gathered}
g_{4}^{(4)} / v_{F}=g_{2}^{(4)} / v_{F}=g_{4}^{(2)} / v_{F}=g_{2}^{(2)} / v_{F} \approx 100 / \kappa, \\
g^{(4)} / v_{F}=g^{(2)} / v_{F}=f^{(4)} / v_{F}=f^{(2)} / v_{F} \approx 100 / \kappa,
\end{gathered}
$$




$$
\begin{gathered}
g_{4}^{(1)} / v_{F}=g_{2}^{(1)} / v_{F} \approx-0.6+3.6 / \kappa, \\
g^{(1)} / v_{F} \approx-1.1+1.1 / \kappa, \\
f^{(1)} / v_{F}=u_{B} / v_{F} \approx-0.5+0.5 / \kappa, \\
u_{F} / v_{F} \approx-0.5+0.3 / \kappa, \\
g_{1}^{(1)} / v_{F}=g_{1}^{(2)} / v_{F} \approx-0.5+0.05 / \kappa .
\end{gathered}
$$

We note again that the different contributions from the Coulomb interaction (with a $1 / \kappa$ reduction in each case) correspond to the pertinent choices of the momentum transfer in the evaluation of $\tilde{V}_{C}(k, q)$.

The relevance of the backscattering interactions comes from the fact that they give rise to quantum corrections that depend logarithmically on the energy scale. By following the renormalization group program, we can translate the logarithmic dependence on energy of the terms in the diagrammatic expansion into the scale dependence of renormalized coupling constants. ${ }^{23}$ Scaling equations for the $g_{i}^{(j)}$ couplings have been written already for a model with two subbands in Ref. 24. Here, we have completed the set of equations and incorporated a nonperturbative improvement by writing the exact dependence of the anomalous dimensions on the forward-scattering couplings through the $K_{+}, K_{-}$, and $K_{0}$ parameters. This is essential to deal with the strong-coupling regime of the forward-scattering interactions in the case of single small-diameter nanotubes. Up to terms quadratic in the backscattering interactions, we find the complete set of scaling equations

$$
\begin{gathered}
\frac{\partial g_{1}^{(1)}}{\partial l}=-\frac{1}{\pi v_{F}}\left(g_{1}^{(1)} g_{1}^{(1)}+g_{1}^{(2)} g_{2}^{(1)}\right)-\frac{1}{\pi v_{F}} \beta u_{F} u_{B} \\
\frac{\partial g_{1}^{(2)}}{\partial l}=\left(1-\frac{1}{K_{-}}\right) g_{1}^{(2)}-\frac{1}{\pi v_{F}}\left[g_{2}^{(1)} g_{1}^{(1)}+(\beta / 2)\left(u_{F}^{2}+u_{B}^{2}\right)\right] \\
\frac{\partial g_{2}^{(1)}}{\partial l}=\left(1-\frac{1}{K_{-}}\right) g_{2}^{(1)}-\frac{1}{\pi v_{F}}\left(2 g_{4}^{(1)} g_{2}^{(1)}-g_{4}^{(1)} g_{1}^{(2)}+g_{1}^{(2)} g_{1}^{(1)}\right. \\
\left.+\beta u_{F} u_{B}\right), \\
\frac{\partial g_{2}^{(2)}}{\partial l}=-\frac{1}{2 \pi v_{F}}\left(g_{2}^{(1)} g_{2}^{(1)}+g_{1}^{(1)} g_{1}^{(1)}+g_{1}^{(2)} g_{1}^{(2)}\right) \\
\frac{\partial g_{4}^{(1)}}{\partial l}=-\frac{1}{\pi v_{F}}\left(g_{4}^{(1)} g_{4}^{(1)}+g_{2}^{(1)} g_{2}^{(1)}-g_{1}^{(2)} g_{2}^{(1)}\right) \\
\frac{\partial g^{(2)}}{\partial l}=-\frac{1}{\pi v_{F}}\left[(\beta / 2) g^{(1)} g^{(1)}+u_{F}^{2}+u_{B}^{2}\right] \\
\frac{\partial l}{2 \pi v_{F}}\left(g_{4}^{(1)} g_{4}^{(1)}-g_{1}^{(2)} g_{1}^{(2)}\right)
\end{gathered}
$$

$$
\begin{gathered}
\frac{\partial g^{(1)}}{\partial l}=-\frac{1}{\pi v_{F}}\left(\beta g^{(1)} g^{(1)}+2 u_{F} u_{B}\right), \\
\frac{\partial f^{(2)}}{\partial l}=-\frac{\alpha}{2 \pi v_{F}}\left(f^{(1)} f^{(1)}-u_{F}^{2}\right), \\
\frac{\partial f^{(1)}}{\partial l}=-\frac{\alpha}{\pi v_{F}}\left(f^{(1)} f^{(1)}+u_{B}^{2}-u_{F} u_{B}\right),
\end{gathered}
$$$$
\frac{\partial u_{F}}{\partial l}=\Delta u_{F}-\frac{1}{2 \pi v_{F}}\left[g_{1}^{(2)} u_{F}+\left(g_{2}^{(1)}+g_{1}^{(1)}+\beta g^{(1)}\right) u_{B}\right]
$$

$$
\begin{aligned}
\frac{\partial u_{B}}{\partial l}= & \Delta u_{B}-\frac{1}{2 \pi v_{F}}\left[g_{1}^{(2)} u_{B}+\left(g_{2}^{(1)}+g_{1}^{(1)}+\beta g^{(1)}\right) u_{F}\right]+\frac{\alpha}{\pi v_{F}} f^{(1)} \\
& \times\left(u_{F}-2 u_{B}\right)
\end{aligned}
$$

where $\beta=v_{F} / v_{F}^{(0)}, \alpha=2 /\left(1+v_{F}^{(0)} / v_{F}\right)$, and the anomalous dimension $\Delta$ depends on $K_{+}, K_{-}, K_{0}$, and $f^{(2)}$ as shown in Appendix A. In the above equations, the variable $l$ stands for minus the logarithm of the energy (temperature) scale measured in units of the high-energy scale $E_{c}$ of the 1D model (of the order of $\sim 0.1 \mathrm{eV}$ ).

We have to stress that the shape of the low-energy subbands, shown schematically in Fig. 2, places a limitation in the range of high energies over which the scaling equations may be integrated. We recall that the flow of the scaling equations represents the progressive integration of electron modes from a strip stretching from the upper cutoff at energy $E_{c}$ to the lower cutoff at $-E_{c}$. In the context of the band structure represented in Fig. 2, it is clear that the upper cutoff has to fall below the top of the degenerate subbands, which places a limit on the range of high-energy states from which to start the integration. In the undoped system, the distance from the Fermi level to the top of the degenerate subbands is $\approx 0.2 \mathrm{eV}$. In this case, there is enough room anyway to describe the flow towards electronic instabilities that are expected to arise at much lower energy scales.

The strong curvature near the top of the degenerate subbands also implies that the slope of the dispersion does not remain constant when integrating the electron modes from the high-energy cutoff down to the Fermi level. In these circumstances, the influence of this drift on the velocity of the excitations must be elucidated when integrating the scaling equations. The most physical approach to address this question is to study the variation of the scaling flow upon shifts in the position of the Fermi level and, consequently, in the Fermi velocity $v_{F}$. This amounts to studying the influence of doping in the electron system. Thus, as well as for the undoped system, we have solved the scaling equations for a doping range up to $6 \%$ of hole doping, where the Fermi velocity $v_{F}$ is already very close to the asymptotic maximum value for the degenerate subbands. We have been able also to find a consistent flow for doping rates of up to 0.02 of electron doping, where the upper cutoff is still below the top of the degenerate subbands (with a reasonable choice of the high-energy scale $\left.E_{c} \approx 0.1 \mathrm{eV}\right)$. Although the analysis of 


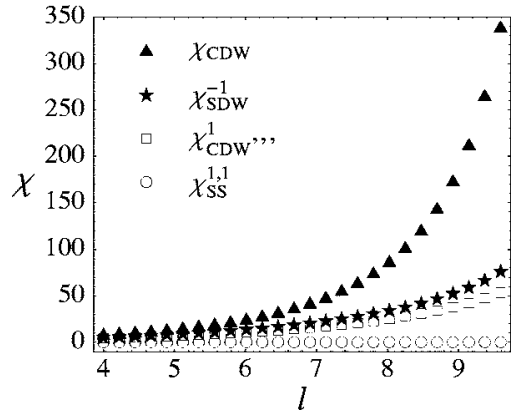

FIG. 4. Flow of the largest response functions (plus $\chi_{S S}^{1,1}$ ) at $\kappa$ $=1.4$.

larger rates of electron doping becomes unfeasible within the renormalization group approach, the range of doping covered is already appropriate to estimate the effects that can be expected from the curvature of the degenerate subbands. We also have to point out that the physically relevant conditions correspond in most cases to the hole doping of the carbon nanotubes, due to either charge transfer to the substrate or from the adsorption of gas molecules.

In order to determine first the electronic instabilities that may appear in single undoped nanotubes, we have solved the set of scaling equations (28)-(39), taking initial values for the couplings according to the above discussion. The essential point is that the scaling equations reflect the strong screening that the couplings undergo at low energies, which leads them to a regime of large attraction. To find the dominant instability in the electron system, one has to look at the behavior of the different response functions $\chi$, which are catalogued in Appendix B. The regime of attraction leads to the large growth of some of the $\chi$; this points at a tendency towards long-range order that cannot be completed anyhow at any finite frequency in the $1 \mathrm{D}$ system. The scaling of the interactions is cut off at some low-energy scale $\omega_{c}$ for which one of the charge stiffnesses $\beta, \mu$, or $K_{-}$diverges. We characterize the dominant instability by identifying the response function that reaches the largest value at the scale $\omega_{c}$.

By varying the dielectric constant, we have found two different regimes, namely a regime characterized by the divergence of $K_{-}$(for $1<\kappa<1.6$ and $\kappa>3.4$ ), and a regime with the divergence of $\beta$ (for $1.6<\kappa<3.4$ ). The largest growth for the response functions corresponds to $\chi_{C D W}$, with large momentum connecting opposite Fermi points of the degenerate subbands (see Appendix B). We can understand this finding by looking at the competition between the phonon exchange and Coulomb contributions in the different channels. At small $\kappa$, the couplings for large transverse momentum transfer $\left(g_{1}^{(1)}, g_{1}^{(2)}\right)$ are attractive from the very beginning [see Eq. (27)]. In particular, the flow is dominated by $g_{1}^{(1)}$, which is driven to large attraction, leading to a rapid growth of $\chi_{C D W}$.

Regardless, as illustrated in Fig. 4, the susceptibility $\chi_{C D W}$ grows large only for values of the dielectric constant close to 1. The values $\kappa \sim 1.4-2$, corresponding to nanotubes in typical conditions, are actually in the crossover to the regime with very small correlations. For $\kappa>2$, the maximum value for all the response functions is $\ll 100$, signaling that the

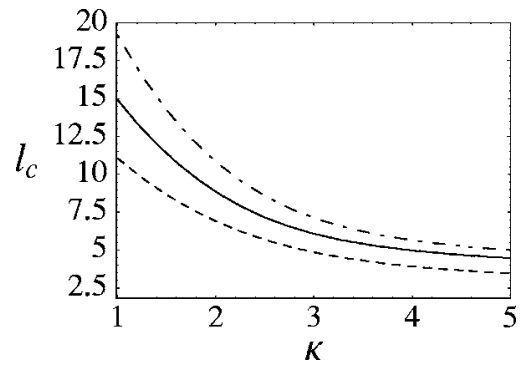

FIG. 5. Plot of minus the logarithm of the energy scale $l_{c}$ $=\ln \left(E_{c} / \omega_{c}\right)$ at which the flow breaks down, as a function of $\kappa$. The full line represents the values for the undoped system, while the dashed (dot-dashed) line corresponds to the case of electron (hole) doping mentioned in the text.

tendency to ordering is weak. We also observe from the comparison in Fig. 4 that, while some charge and spin-densitywave response functions grow large by approaching the critical value $l_{c}$ (at least for small $\kappa$ ), the superconducting response functions for different order parameters all remain small. This finding seems to rule out the possibility of having superconducting correlations in the $(5,0)$ nanotubes, at least under the physical conditions considered in the present section.

In Fig. 5 we have plotted the critical value $l_{c}=\ln \left(E_{c} / \omega_{c}\right)$ at which the flow breaks down as a function of $\kappa$. It appears that the energy scale $\omega_{c}$ depends significantly on the value of the dielectric constant, implying that the onset of the electronic instability can be found at temperatures ranging from $T_{c} \sim 10^{-4} \mathrm{~K}($ at $\kappa \approx 1)$ up to a value $T_{c} \sim 10^{2} \mathrm{~K}$ (reached from $\kappa \approx 10$ ).

The scale $l_{c}$ also becomes quite sensitive to changes in the Fermi level of the system. As mentioned before, we have considered the case of hole doping, reaching doping rates $\delta$ down to -0.06 for which the Fermi velocity $v_{F}$ is already very close to the asymptotic slope in the dispersion of the degenerate subbands. In that instance, the reduction in the density of states leads to a significant decrease in the energy scale for the breakdown of Luttinger liquid behavior, as shown in Fig. 5. Within the limitations pointed out before, we have also analyzed the influence of electron doping in the system. For doping rates $\delta \approx 0.02$, the critical scale $l_{c}$ is modified in the way represented in Fig. 5. We observe that the effect of electron doping leads to a significant increase of the energy scale $\omega_{c}$, which is particularly strong for small values of the dielectric constant.

The inspection of the different instabilities that arise at $\omega_{c}$ upon doping is also interesting. The results can be summarized by making a map of the different low-energy phases under variations in the doping rate $\delta$ and the dielectric constant $\kappa$. The phase diagram is represented in Fig. 6. We observe that the phase corresponding to $\beta^{-1}=0$ shrinks by electron doping, while another phase with $\mu^{-1}=0$ appears by hole doping near the physically relevant region around $\kappa$ $\approx 1.4$. We arrive in this way at a continuous picture of the trend from hole- to electron doping, making the catalogue of the different types of Luttinger liquid breakdown arising at low temperatures in single $(5,0)$ nanotubes. 


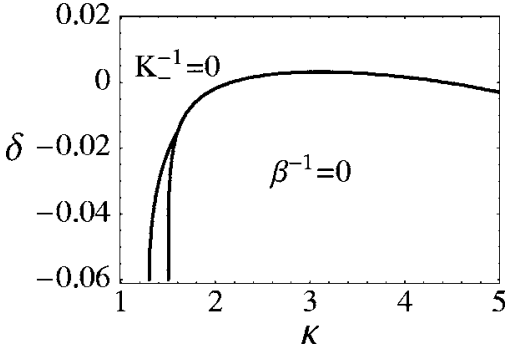

FIG. 6. Phase diagram showing the different low-energy instabilities of single $(5,0)$ nanotubes, depending on the doping rate $\delta$ and the dielectric constant $\kappa$. The narrow phase to the left of the diagram corresponds to the divergence of the $\mu$ parameter.

\section{INTERTUBE SCREENING AND INSTABILITIES IN A 3D ARRAY OF NANOTUBES}

While the above framework provides an accurate description of the instabilities of single small-diameter nanotubes, the analysis has to be conveniently modified when the electron system is composed of a manifold of nanotubes. This is the situation in the experiments reported in Ref. 20, where the samples contain large 3D arrays of small-diameter nanotubes. Systems that are formed by the assembly of a large amount of nanotubes, as is also the case of nanotube ropes, require in general an additional analysis of the interactions arising between different nanotubes. We will now focus on the conditions of the experimental samples studied in Ref. 20 , which present a negligible intertube tunneling but have a significant coupling between electronic currents in different nanotubes.

Specifically, we will consider the case of an array of small-diameter nanotubes, arranged as a triangular lattice as viewed from a cross section of the 3D array. We will denote by $d$ the distance between nearest-neighbor nanotubes in such a lattice, having in mind that, for the samples studied in Ref. 20, its value is given by $d \approx 1 \mathrm{~nm}$. The coupling between different nanotubes at positions $\mathbf{I}$ and $\mathbf{I}^{\prime}$ (measured in the cross section of the array) comes from the Coulomb interaction, due to its long-range character. It may couple currents with large transverse separation $\left|\mathbf{I}-\mathbf{l}^{\prime}\right|$ as long as the longitudinal momentum transfer $k$ becomes as small as $\left|\mathbf{I}-\mathbf{I}^{\prime}\right|^{-1}$. The Coulomb potential $V_{\mathbf{l}, \mathbf{I}^{\prime}}(k)$ between currents in different nanotubes $\mathbf{I}$ and $\mathbf{I}^{\prime}$ is actually obtained by partially Fourier transforming the 3D Coulomb potential in the longitudinal direction

$$
V_{1,1^{\prime}}(k) \approx \frac{2 e^{2}}{\kappa} K_{0}\left(\left|\mathbf{I}-\mathbf{l}^{\prime}\right| k\right),
$$

$K_{0}(x)$ being the modified Bessel function, which diverges logarithmically as $x \rightarrow 0$ and is exponentially suppressed as for $x>1$.

It is clear then that the nanotubes in the array may screen efficiently the forward-scattering interactions within each nanotube. This effect can be studied by extending the random-phase approximation (RPA) scheme to incorporate the electrostatic coupling between all the nanotubes in the array, as described in Appendix C. The main conclusion of

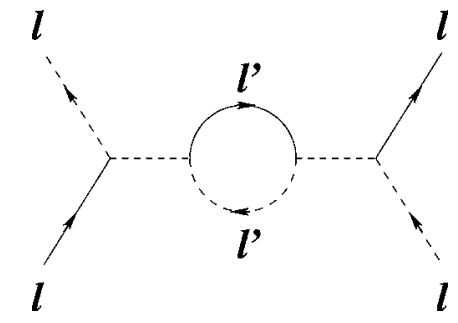

FIG. 7. Second-order process with logarithmic dependence on the frequency renormalizing intraband interactions at a given nanotube $\mathbf{l}$ through the coupling with the nearest neighbors $\mathbf{I}^{\prime}$ in a $3 \mathrm{D}$ array of nanotubes. The full (dashed) lines with an arrow represent the propagation of electrons with right (left) chirality, and the dashed lines without arrow stand for the Coulomb interaction between electronic currents in nearest-neighbor nanotubes.

this study is that the screened Coulomb potential $V_{1,1}^{(r)}(k)$ within each nanotube becomes finite in the limit of vanishing momentum $k \rightarrow 0$, reaching a saturation value $V_{\mathbf{l}, \mathbf{l}}^{(r)}(k=0)$ $\approx 0.08 e^{2}$. This corresponds to a dimensionless coupling $V_{1.1}^{(r)}$ $\times(k=0) / v_{F} \approx 0.65$, which is about two orders of magnitude smaller than the one for single nanotubes. In this case the phonon-exchange contribution competes with the Coulomb repulsion also in the intratube forward-scattering channels, changing qualitatively the physical picture.

One more important effect is that the coupling between currents in neighboring nanotubes may also correct the intratube backscattering interactions with small momentum transfer. This is due to the fact that, for a longitudinal momentum transfer $2 k_{F}$, the Coulomb potential between nearest-neighbor nanotubes (such that $\left|\mathbf{I}-\mathbf{l}^{\prime}\right|=d$ ) still has a non-negligible strength, $V_{1, I^{\prime}}\left(2 k_{F}\right) \approx 0.037 e^{2}$. The important point is that this interaction gives rise to processes of the type depicted in Fig. 7, which depend on the energy scale and therefore introduce important renormalization effects for the intratube interactions at low energies. As observed from Fig. 7, the scaling analysis requires the definition of new backscattering interactions that couple electron currents in different nanotubes. We will assign the couplings $\widetilde{g}_{2}^{(1)}$ and $\widetilde{g}_{4}^{(1)}$ to the new channels represented in Figs. 8(a) and $8(\mathrm{~b})$. As we are going to see next, these new couplings mix upon renormalization with two more channels corresponding to forward-scattering interactions between currents with different chirality in nearest-neighbor nanotubes. These will be labeled by the couplings $\widetilde{g}_{4}^{(2)}$ and $\widetilde{g}_{2}^{(2)}$, as shown in Figs. 8(c) and $8(\mathrm{~d})$.

The main role of the intertube backscattering interactions $\widetilde{g}_{4}^{(1)}$ and $\widetilde{g}_{2}^{(1)}$ is to screen the intratube backscattering interactions through processes of the type shown in Fig. 7. Thus, the scaling equations for $g_{2}^{(1)}$ and $g_{4}^{(1)}$ get new contributions in the $3 \mathrm{D}$ array of nanotubes, taking now the form

$$
\begin{aligned}
\frac{\partial g_{2}^{(1)}}{\partial l}= & \left(1-\frac{1}{K_{-}}\right) g_{2}^{(1)}-\frac{1}{\pi v_{F}}\left(2 g_{4}^{(1)} g_{2}^{(1)}-g_{4}^{(1)} g_{1}^{(2)}+g_{1}^{(2)} g_{1}^{(1)}\right. \\
& \left.+\beta u_{F} u_{B}+12 \widetilde{g}_{4}^{(1)} \widetilde{g}_{2}^{(1)}\right)
\end{aligned}
$$




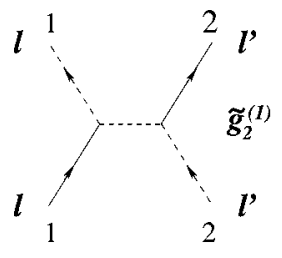

(a)

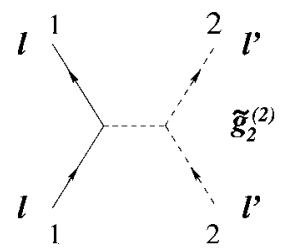

(c)

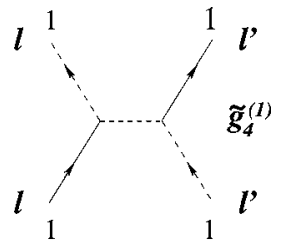

(b)

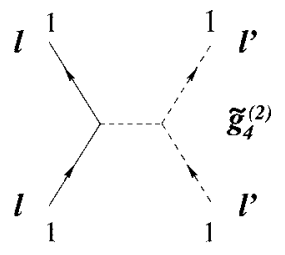

(d)
FIG. 8. Intertube interactions arising from the coupling between electronic currents in nearest-neighbor nanotubes $\mathbf{l}$ and $\mathbf{l}^{\prime}$ of a 3D array. The meaning of the different lines is the same as in Fig. 7.

$$
\begin{aligned}
\frac{\partial g_{4}^{(1)}}{\partial l}= & -\frac{1}{\pi v_{F}}\left(g_{4}^{(1)} g_{4}^{(1)}+g_{2}^{(1)} g_{2}^{(1)}-g_{1}^{(2)} g_{2}^{(1)}+6 \widetilde{g}_{4}^{(1)} \widetilde{g}_{4}^{(1)}\right. \\
& \left.+6 \widetilde{g}_{2}^{(1)} \widetilde{g}_{2}^{(1)}\right) .
\end{aligned}
$$

We observe that the new terms are enhanced by a factor proportional to the number of nearest neighbors of each nanotube in the 3D array. On the other hand, the scaling equations for the new intertube couplings turn out to be, to second order in the couplings

$$
\begin{aligned}
& \frac{\partial \widetilde{g}_{2}^{(1)}}{\partial l}=-\frac{1}{\pi v_{F}}\left(2 g_{4}^{(1)} \widetilde{g}_{2}^{(1)}+2 g_{2}^{(1)} \widetilde{g}_{4}^{(1)}+4 \widetilde{g}_{4}^{(1)} \widetilde{g}_{2}^{(1)}+\widetilde{g}_{2}^{(2)} \widetilde{g}_{2}^{(1)}\right. \\
&\left.-g_{4}^{(2)} \widetilde{g}_{2}^{(1)}-g_{1}^{(2)} \widetilde{g}_{4}^{(1)}\right) \\
& \frac{\partial \widetilde{g}_{4}^{(1)}}{\partial l}=-\frac{1}{\pi v_{F}}\left(2 g_{4}^{(1)} \widetilde{g}_{4}^{(1)}+2 g_{2}^{(1)} \widetilde{g}_{2}^{(1)}+2 \widetilde{g}_{4}^{(1)} \widetilde{g}_{4}^{(1)}+2 \widetilde{g}_{2}^{(1)} \widetilde{g}_{2}^{(1)}\right. \\
&\left.+\widetilde{g}_{4}^{(2)} \widetilde{g}_{4}^{(1)}-g_{4}^{(2)} \widetilde{g}_{4}^{(1)}-g_{1}^{(2)} \widetilde{g}_{2}^{(1)}\right) \\
& \frac{\partial \widetilde{g}_{4}^{(2)}}{\partial l}=-\frac{1}{2 \pi v_{F}} \widetilde{g}_{4}^{(1)} \widetilde{g}_{4}^{(1)} \\
& \frac{\partial \widetilde{g}_{2}^{(2)}}{\partial l}=-\frac{1}{2 \pi v_{F}} \widetilde{g}_{2}^{(1)} \widetilde{g}_{2}^{(1)}
\end{aligned}
$$

Let us now discuss the initial conditions for the new set of scaling equations. The intratube backscattering couplings have the same initial values as in the preceding section since, according to the above discussion, the intertube screening in these channels is already incorporated in the scaling equations. Conversely, in the forward-scattering channels, the screening may be represented by finite diagrammatic corrections, which can be summed up with the RPA approach described in Appendix C. As mentioned before, the Coulomb contribution is given by the dimensionless coupling $\widetilde{V}_{\mathbf{l}, \mathbf{I}}^{(r)}(k$ $=0) / v_{F} \approx 0.65$. The phonon-exchange contribution at vanish-

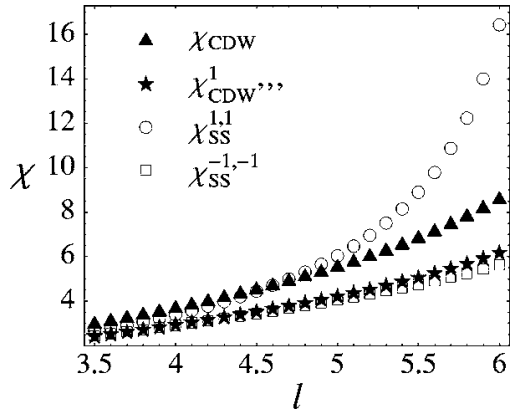

FIG. 9. Flow of the largest response functions at $\kappa=2$.

ing momentum transfer can be estimated to be very similar to the value found from (19), showing that there is now a substantial suppression of the effects of the Coulomb repulsion in the intratube forward-scattering channels.

On the other hand, the intertube forward-scattering interactions are also screened due to the electrostatic coupling among the nanotubes in the array. These screening effects can be evaluated again within the RPA scheme in Appendix $\mathrm{C}$, with the result that they render finite the intertube screened Coulomb potential at $k \rightarrow 0$. Furthermore, the values of the intertube backscattering couplings $\widetilde{g}_{2}^{(1)}$ and $\widetilde{g}_{4}^{(1)}$ can be obtained from (40) with $\left|\mathbf{l}-\mathbf{l}^{\prime}\right|=d$. The new initial conditions obtained in this way read

$$
\begin{aligned}
& \widetilde{g}_{4}^{(2)} / v_{F}=\widetilde{g}_{2}^{(2)} / v_{F} \approx 0.002 \kappa, \\
& \widetilde{g}_{2}^{(1)} / v_{F}=\widetilde{g}_{4}^{(1)} / v_{F} \approx 0.28 / \kappa .
\end{aligned}
$$

We observe that the $\kappa$ dependence of the screened forwardscattering couplings is qualitatively different with respect to the nonscreened interactions. In particular, we find that the intertube couplings have an approximately linear dependence on $\kappa$, at least up to $\kappa \sim 5$ (see Appendix C).

The numerical integration of the scaling equations shows now a new physical regime, where the instability of the system is not characterized by the large growth of any of the response functions, but only by the divergence of the charge stiffness $\beta$. We have plotted in Fig. 9 a typical flow of the dominant response functions up to the energy scale at which the divergence of the charge stiffness occurs. We note that the screening effects produced by the environment of nanotubes suppress the tendency to CDW ordering, and enhance the superconducting correlations. Anyway, the values of $\chi_{S S}^{1,1}$ do not grow large, making it very unlikely that they may give rise to any observable feature.

Otherwise, the divergence of $\beta$ signals the onset of a regime of strong attraction, as it implies the divergence of the compressibility and density-density correlator in the corresponding sector, and the vanishing of the renormalized Fermi velocity $u_{+} / \beta$ as well. This points to the development of phase separation into spatial regions with different electronic density, in close analogy with the physical interpretation of the Wentzel-Bardeen singularity. The critical energy scale for this instability is shown in Fig. 10. For the zeolite matrix of Ref. 20, the estimate of the dielectric constant gives $\kappa$ 


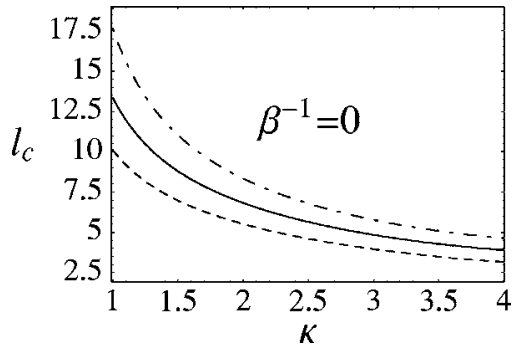

FIG. 10. Plot of minus the logarithm of the energy scale $l_{c}$ $=\ln \left(E_{c} / \omega_{c}\right)$ at which the flow breaks down, as a function of $\kappa$. The full line represents the values for the undoped system, while the dashed (dot-dashed) line corresponds to the case of electron (hole) doping mentioned in the text. The divergent Luttinger liquid parameter is also indicated.

$\approx 2-4$, which corresponds to a transition temperature $T_{c}$ $\approx 3-20 \mathrm{~K}$, in qualitative agreement with the value $T_{c}$ $\approx 15 \mathrm{~K}$ observed experimentally.

We have also studied the effect of doping and the consequent shift in the Fermi level of the carbon nanotubes. We have considered again the range from hole doping, with a doping rate $\delta$ down to -0.06 , to electron doping with $\delta$ up to 0.02 . The variation of the critical scale $l_{c}$ between these two limits is shown in Fig. 10. As in the case of single nanotubes, the instance of hole doping brings about a reduction of the density of states at the Fermi level, and implies a significant decrease of the critical energy $\omega_{c}$. The case of electron doping has the opposite effect reflected in Fig. 10. We have to point out, however, that for large values of $\kappa$ the range of variation of $l_{c}$ becomes rather small. We also remark that the breakdown of the Luttinger liquid regime takes place always through the divergence of the $\beta$ parameter, at least within the above-mentioned range of doping rates.

Finally, we point out that the above results cannot be assimilated to those obtained within the usual two-band model of metallic nanotubes. The main difference is the development of superconducting correlations in the present model, which are completely absent, for instance, in the $(3,3)$ geometry. ${ }^{33}$ This is due to the influence of new couplings, like $u_{F}$ and $u_{B}$, which enhance $\chi_{S S}^{1,1}$ when driven to the attractive regime [see Eq. (B2)]. We also note that the divergence of $\beta$ provides a regime of phase separation into regions with different electronic density, referred to a sector of the model in which the three low-energy subbands are entangled through the coupling $f^{(2)}$, which is a peculiar feature of the three-band model.

\section{CONCLUSION}

In this paper we have studied the electronic instabilities of the small-diameter $(5,0)$ nanotubes, by analyzing the competition between the Coulomb interaction and the effective interaction mediated by the exchange of phonons. We have built our framework on the basis that the Luttinger liquid is what characterizes the normal state of the carbon nanotubes. This is a key assumption, since the low-energy excitations of the 1D electron system are given in the normal state by charge and spin fluctuations, with absence of electron quasi- particles in the spectrum. This is consistent with the powerlaw suppression of the conductance and the differential conductivity that has been measured in several transport experiments on carbon nanotubes. ${ }^{2,3,34}$ The deviations from this picture have to be understood as perturbations of the Luttinger liquid behavior, which in general are nominally small as the strong Coulomb potential at small momentum transfer provides the dominant interaction in the system.

There are however instances in which the enhancement of the backscattering interactions (corresponding to some nesting momentum between Fermi points) leads to the breakdown of the Luttinger liquid picture. This certainly happens at sufficiently small temperature, since the backscattering interactions are amplified at low energies until they reach the strong-coupling regime. ${ }^{23}$ There are also experimental conditions in which the strong Coulomb repulsion is largely screened. The screening effects are particularly important when the nanotubes form large assemblies. ${ }^{35}$ This should explain for instance the appearance of a regime with superconducting correlations at low temperature in massive ropes. In this context, the increase in the curvature of the smalldiameter nanotubes has to give rise to larger electron-phonon couplings, ${ }^{18}$ implying in turn a natural enhancement of the backscattering interactions mediated by phonon exchange. Thus, there have been good prospects to observe the effects of large electron correlations in the small-diameter nanotubes, specially in the $(5,0)$ geometry which leads to a relatively large density of states at the Fermi level.

We have shown that the level of screening of the Coulomb interaction plays an important role in the development of the low-energy instabilities in the $(5,0)$ nanotubes. In the case of single nanotubes exposed to the screening by external gates, the Luttinger liquid is unstable against the onset of a strong-coupling phase with very large CDW correlations. The temperature of crossover to the new phase depends crucially on the dielectric constant $\kappa$ of the environment, ranging from $T_{c} \sim 10^{-4} \mathrm{~K}($ at $\kappa \approx 1)$ up to a value $T_{c} \sim 10^{2} \mathrm{~K}$ (reached from $\kappa \approx 10$ ). The inspection of the response functions measuring the superconducting correlations shows that these do not have an appreciable growth in the regime where the CDW correlations grow large. Thus, even under conditions of strong screening by external charges, the observation of any superconducting feature seems to be excluded in single nanotubes with the $(5,0)$ geometry.

The physical picture is different when we consider the case of a large array of nanotubes, of the kind patterned by the channels of the zeolite matrix in the experiments of Ref. 20. The coupling between the nanotubes in the array leads to a large reduction of the Coulomb potential, which is rendered finite at vanishing momentum transfer. The Coulomb interaction happens now to be in the weak-coupling regime, therefore making the destabilization of the Luttinger liquid easier. We have found however that, before the superconducting correlations may grow large, a singularity is reached in one of the charge stiffnesses of the Luttinger liquid. This implies the divergence of the compressibility in one of the charge sectors, and it has therefore the same physical interpretation as the Wentzel-Bardeen singularity. ${ }^{27}$ As the density-density correlator in each charge sector is proportional to the charge stiffness, the divergence of the latter 
leads to a phase where the system prefers to form electron aggregates, leaving some other regions with a defect of electron density. The possibility of a phase of this type has been anticipated already in Ref. 19. Furthermore, as already stressed in Ref. 33, the divergence of the charge stiffness leads also to the development of a pseudogap in the singleparticle spectrum. We have seen that, for a choice of the dielectric constant in accordance with the experimental conditions described in Ref. 20, the transition temperature found in our analysis is of the same order as the temperature of the crossover to the pseudogap regime observed in that reference.

An important question has to do with the influence of disorder in the $(5,0)$ nanotubes. The effects of impurities in the transport properties depend in general on the position of the Fermi level, and it is known that they may be particularly relevant in the case of massive subbands. This has been confirmed by the results of Ref. 36, where the mean-free path and the conductance have been computed for a model of $(8,0)$ nanotubes with averaged disorder. It has been shown there that the conductance is strongly suppressed right at the energy where a new subband opens in the spectrum. When transposing these results to the low-energy subbands in our undoped system, it turns out however that the distance of the Fermi level to the edge of the degenerate subbands $(\approx 0.2 \mathrm{eV})$ is sufficiently large to discard any drastic influence of disorder on the transport properties. Thus, although the scattering due to disorder could severely modify the density of states near the Van Hove singularity, this would have a relevant influence only under conditions of sufficiently large electron doping in our model. For the more likely experimental conditions of slight hole doping, the effects of disorder have to be much less relevant at energies around the Fermi level.

The relative weakness of those effects is consistent with the experimental observations of transport properties reported in Ref. 20. The measures of the conductivity in very thin samples seem to imply ballistic transport over length scales at least of the order of $\sim 50 \mathrm{~nm}$. Moreover, the value quoted for the conductivity at room temperature suggests that the electron system is not in the localization regime, in samples with very large thickness of the order of $\sim 100 \mu \mathrm{m}$.

There are however experimental features of the smalldiameter nanotubes described in Ref. 20 that cannot be explained in terms of the electronic instabilities we have found in the present paper. The strong diamagnetic signal measured at low temperatures has been interpreted in that reference as a consequence of entering a superconducting regime in the small-diameter nanotubes. The question of whether the armchair $(3,3)$ nanotubes can be responsible for such a regime, or for some other type of electronic instability that may account for the strong diamagnetic signal, as some preliminary results seem to indicate, ${ }^{33}$ remains open. Other questions that deserve attention refer to the properties of the small-diameter nanotubes when eventually assembled into other aggregates different from those considered in the present paper. It may be interesting to see the influence of intertube tunneling between small-diameter nanotubes packed to form massive ropes. It will be crucial then to discern whether the zigzag $(5,0)$ and the armchair $(3,3)$ nanotubes lead to different phases, and whether they may support the appearance of larger superconducting correlations than those observed in massive ropes made of typical nanotubes.

\section{ACKNOWLEDGMENTS}

The financial support of the Ministerio de Educación y Ciencia (Spain) through Grant BFM2003-05317 is gratefully acknowledged. E. P. was also supported by INFN Grant 10068.

\section{APPENDIX A: DIAGONALIZATION OF $H_{F S}$ AND EVALUATION OF THE ANOMALOUS DIMENSIONS}

In this appendix we give the results of diagonalizing $H_{F S}$ by means of a generalized Bogoliubov transformation. Since in Eq. (11) the $\left\{\Phi_{-}, \Pi_{-}\right\}$sector is already decoupled, it is sufficient to operate the transformation by mixing only the $\widetilde{\Phi}_{+}, \widetilde{\Phi}_{0}$ fields and their corresponding conjugate momenta $\widetilde{\Pi}_{+}, \widetilde{\Pi}_{0}$. Therefore, we introduce the new operators $\hat{\Phi}_{+}, \hat{\Phi}_{0}, \hat{\Pi}_{+}, \hat{\Pi}_{0}$ defined by

$$
\begin{aligned}
& \widetilde{\Phi}_{0} \equiv c_{0} \sqrt{\mu} \hat{\Phi}_{0}+s_{0} \sqrt{\nu} \hat{\Phi}_{+}, \quad \widetilde{\Pi}_{0} \equiv \frac{c_{0}}{\sqrt{\mu}} \hat{\Pi}_{0}+\frac{s_{0}}{\sqrt{\nu}} \hat{\Pi}_{+}, \\
& \widetilde{\Phi}_{+} \equiv c_{+} \sqrt{\beta} \hat{\Phi}_{+}+s_{+} \sqrt{\alpha} \hat{\Phi}_{0}, \quad \widetilde{\Pi}_{+} \equiv \frac{c_{+}}{\sqrt{\beta}} \hat{\Pi}_{+}+\frac{s_{+}}{\sqrt{\alpha}} \hat{\Pi}_{0},
\end{aligned}
$$

where $c_{0(+)} \equiv \cos \varphi_{0(+)}$ and $s_{0(+)} \equiv \sin \varphi_{0(+)}$ in order to ensure the standard commutation relations $\left[\hat{\Phi}_{0(+)}(x), \hat{\Pi}\left(x^{\prime}\right)_{0(+)}\right]$ $=i \delta\left(x-x^{\prime}\right)$.

The angles $\varphi_{0}, \varphi_{+}$and the parameters $\mu, \nu, \alpha, \beta$ depend on $K_{0}, K_{+}, u_{0}, u_{+}, f^{(2)}, f^{(4)}$, and must be determined by imposing that (i) $\left[\hat{\Phi}(x)_{0(+)}, \hat{\Pi}\left(x^{\prime}\right)_{+(0)}\right]=0$; (ii) $H_{F S}$ written in terms of the new fields gets diagonal; (iii) the coefficients of $\left(\partial_{x} \hat{\Phi}_{0(+)}\right)^{2}$ and $\left(\hat{\Pi}_{0(+)}\right)^{2}$ are the same. The corresponding algebraic system has an analytical solution in closed form under the condition $f^{(2)}=f^{(4)}$ (Ref. 37)

$$
\begin{gathered}
\varphi_{0}=\frac{1}{2} \arctan \left(\frac{4 \sqrt{2 K_{0} K_{+}} f^{(2)} / \pi}{u_{+}^{3 / 2} / u_{0}^{1 / 2}-u_{0}^{3 / 2} / u_{+}^{1 / 2}}\right), \\
\varphi_{+}=-\varphi_{0}, \\
\mu=\left(c_{0}^{2}+s_{0}^{2} u_{+}^{2} / u_{0}^{2}-4 \sqrt{2 K_{0} K_{+}} f^{(2)} c_{0} s_{0} u_{+}^{1 / 2} / \pi u_{0}^{3 / 2}\right)^{-1 / 2}, \\
\beta=\left(c_{0}^{2}+s_{0}^{2} u_{0}^{2} / u_{+}^{2}+4 \sqrt{2 K_{0} K_{+}} f^{(2)} c_{0} s_{0} u_{0}^{1 / 2} / \pi u_{+}^{3 / 2}\right)^{-1 / 2}, \\
\alpha=\mu u_{+} / u_{0}, \\
\nu=\beta u_{0} / u_{+} .
\end{gathered}
$$

Upon the complete Bogoliubov transformation, the renormalized velocities of the new free-boson Hamiltonian become 


$$
\begin{aligned}
& u_{+} \rightarrow u_{+} / \beta, \\
& u_{0} \rightarrow u_{0} / \nu .
\end{aligned}
$$

The above solution can be used to calculate the nonperturbative contribution $\Delta$ to the scaling equations of $u_{F}$ and $u_{B}$. The quantity $\Delta$ is nothing but the anomalous dimension coming from the forward-scattering interactions. The operators introduced by $u_{F}$ and $u_{B}$ in the Hamiltonian actually can be expressed in terms of the fields in (11) by means of bosonization techniques. This leads to the result

$$
\begin{aligned}
\Delta= & 1-\left(\frac{c_{0}}{\sqrt{2 K_{0} \mu}}+\frac{s_{0}}{2 \sqrt{K_{+} \alpha}}\right)^{2}-\left(\frac{s_{0}}{\sqrt{2 K_{0} \nu}}-\frac{c_{0}}{2 \sqrt{K_{+} \beta}}\right)^{2} \\
& -\left(\frac{1}{2 \sqrt{K_{-}}}\right)^{2} .
\end{aligned}
$$

By means of the same techniques, we can compute also the anomalous dimensions of the operators $O_{\mu}^{P, Q}$ defining the two-particle correlation functions, introduced in Appendix B. They encode the nonperturbative corrections to the scaling equations (B2) for the response functions $\chi$, and read

$$
\begin{aligned}
& \Delta_{D W}=1-\frac{K_{-}}{2}-\frac{K_{+}}{2}\left(c_{0}^{2} \beta+s_{0}^{2} \alpha\right), \\
& \Delta_{D W^{\prime}}=1-\frac{1}{2 K_{-}}-\frac{K_{+}}{2}\left(c_{0}^{2} \mu+s_{0}^{2} \nu\right), \\
& \Delta_{D W^{\prime \prime}}=1-K_{0}\left(c_{0}^{2} \mu+s_{0}^{2} \nu\right), \\
& \Delta_{D W^{\prime \prime \prime}}=1-\frac{K_{-}}{8}-\frac{1}{8 K_{-}}-2\left(\frac{\sqrt{K_{+} \beta} c_{0}}{4}+\frac{\sqrt{K_{0} \nu} s_{0}}{2 \sqrt{2}}\right)^{2} \\
& -2\left(\frac{\sqrt{K_{0} \mu} c_{0}}{2 \sqrt{2}}-\frac{\sqrt{K_{+} \alpha} s_{0}}{4}\right)^{2}-2\left(\frac{c_{0}}{4 \sqrt{K_{+} \beta}}\right. \\
& \left.-\frac{s_{0}}{2 \sqrt{2} \sqrt{K_{0} \nu}}\right)^{2}-2\left(\frac{s_{0}}{4 \sqrt{K_{+} \alpha}}+\frac{c_{0}}{2 \sqrt{2} \sqrt{K_{0} \mu}}\right)^{2}, \\
& \Delta_{S C}^{(a)}=1-\frac{1}{2 K_{-}}-\frac{1}{2 K_{+}}\left(\frac{c_{0}^{2}}{\beta}+\frac{s_{0}^{2}}{\alpha}\right), \\
& \Delta_{S C}^{(b)}=1-\frac{1}{K_{0}}\left(\frac{c_{0}^{2}}{\mu}+\frac{s_{0}^{2}}{\nu}\right) \text {, } \\
& \Delta_{S C^{\prime}}=1-\frac{K_{-}}{2}-\frac{1}{2 K_{+}}\left(\frac{c_{0}^{2}}{\mu}+\frac{s_{0}^{2}}{\nu}\right) \\
& \Delta_{S C^{\prime \prime}}=1-\frac{K_{-}}{8}-\frac{1}{8 K_{-}}-2\left(\frac{\sqrt{K_{+} \beta} c_{0}}{4}-\frac{\sqrt{K_{0} \nu} s_{0}}{2 \sqrt{2}}\right)^{2}-2\left(\frac{\sqrt{K_{0} \mu} c_{0}}{2 \sqrt{2}}\right. \\
& \left.+\frac{\sqrt{K_{+} \alpha} s_{0}}{4}\right)^{2}-2\left(\frac{c_{0}}{4 \sqrt{K_{+} \beta}}+\frac{s_{0}}{2 \sqrt{2} \sqrt{K_{0} \nu}}\right)^{2}-2\left(\frac{s_{0}}{4 \sqrt{K_{+} \alpha}}\right. \\
& \left.-\frac{c_{0}}{2 \sqrt{2} \sqrt{K_{0} \mu}}\right)^{2} \text {. }
\end{aligned}
$$

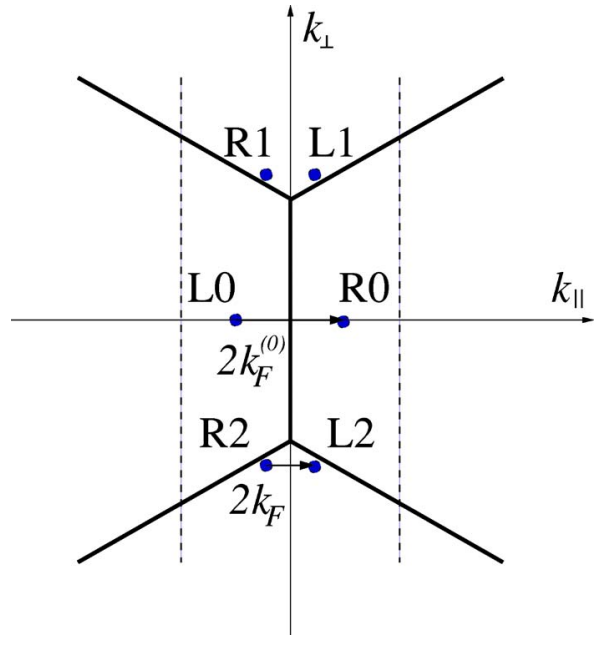

FIG. 11. (Color online) Sketch of the Brillouin zone of the $(5,0)$ nanotube: the six Fermi points are indicated with the labels of corresponding chirality and subband. The longitudinal momentum transfers $2 k_{F}$ and $2 k_{F}^{(0)}$ are also drawn.

Finally, we give also the results for the anomalous dimensions $\alpha_{i}$ governing the density of states near the Fermi level for electrons in the different subbands $i=0,1,2$ in the Luttinger liquid regime

$$
\begin{gathered}
\alpha_{1}=\alpha_{2}=\frac{1}{8}\left[K_{+}\left(c_{0}^{2} \beta+s_{0}^{2} \alpha\right)+\frac{1}{K_{+}}\left(\frac{c_{0}^{2}}{\beta}+\frac{s_{0}^{2}}{\alpha}\right)+K_{-}+\frac{1}{K_{-}}-4\right], \\
\alpha_{0}=\frac{1}{4}\left[K_{0}\left(c_{0}^{2} \mu+s_{0}^{2} \nu\right)+\frac{1}{K_{0}}\left(\frac{c_{0}^{2}}{\mu}+\frac{s_{0}^{2}}{\nu}\right)-2\right] .
\end{gathered}
$$

\section{APPENDIX B: SCALING EQUATIONS FOR THE RESPONSE FUNCTIONS}

In order to study the competition between the possible instabilities, one has to consider the scaling flow of the twoparticle correlation functions $\chi$. They measure the strength of the quantum fluctuations for a given type of ordering induced by the pair field $O_{\mu}^{P, Q}$, in such a way that

$$
\begin{aligned}
\chi_{\mu}^{P, Q}\left(k, \omega_{m}\right)= & -\int_{0}^{\beta} \int_{0}^{L} d \tau d x e^{i k x-i \omega_{m} \tau} \\
& \times\left\langle O_{\mu}^{P, Q}(x, \tau)^{\dagger} O_{\mu}^{P, Q}(0,0)\right\rangle,
\end{aligned}
$$

where $L$ is the length of the nanotube, $\omega_{m}$ is the bosonic Matsubara frequency, and $P, Q= \pm 1$ take into account the band entanglement. ${ }^{38}$ By neglecting the dependence on $\omega_{m}$ and expressing the momenta according to Fig. 11, the complete collection of the Fourier transforms of the response functions reads

$$
\begin{aligned}
O_{D W, \mu}^{P}\left(k \approx 2 k_{F_{L 2}}\right)= & \frac{1}{2 \sqrt{L}} \sum_{p, \alpha, \beta}\left[\Psi_{R 1 \alpha}^{\dagger}(p-k) \sigma_{\mu}^{\alpha, \beta} \Psi_{L 2 \beta}(p)\right. \\
& \left.+P \Psi_{L 1 \alpha}^{\dagger}(p-k) \sigma_{\mu}^{\alpha, \beta} \Psi_{R 2 \beta}(p)\right]
\end{aligned}
$$




$$
\begin{aligned}
& O_{D W^{\prime}, \mu}^{P}\left(k \approx 2 k_{F}\right)=\frac{1}{2 \sqrt{L}} \sum_{p, \alpha, \beta}\left[\Psi_{R 1 \alpha}^{\dagger}(p-k) \sigma_{\mu}^{\alpha, \beta} \Psi_{L 1 \beta}(p)\right. \\
& \left.+P \Psi_{R 2 \alpha}^{\dagger}(p-k) \sigma_{\mu}^{\alpha, \beta} \Psi_{L 2 \beta}(p)\right], \\
& O_{D W^{\prime \prime}, \mu}\left(k \approx 2 k_{F}^{(0)}\right)=\frac{1}{\sqrt{L}} \sum_{p, \alpha, \beta}\left[\Psi_{R 0 \alpha}^{\dagger}(p-k) \sigma_{\mu}^{\alpha, \beta} \Psi_{L 0 \beta}(p)\right], \\
& O_{D W^{\prime \prime \prime}, \mu}^{P}\left(k \approx k_{F_{L 0}}-k_{F_{R 1}}\right)=\frac{1}{2 \sqrt{L}} \sum_{p, \alpha, \beta}\left[\Psi_{R 1 \alpha}^{\dagger}(p\right. \\
& -k) \sigma_{\mu}^{\alpha, \beta} \Psi_{L 0 \beta}(p) \\
& \left.+P \Psi_{R 0 \alpha}^{\dagger}(p-k) \sigma_{\mu}^{\alpha, \beta} \Psi_{L 2 \beta}(p)\right], \\
& O_{S C, \mu}^{P, Q}(k \approx 0)=\frac{1}{\sqrt{3 L}} \sum_{p, \alpha, \beta} \alpha\left[\Psi_{R 1 \alpha}(-p+k) \sigma_{\mu}^{-\alpha, \beta} \Psi_{L 2 \beta}(p)\right. \\
& +P \Psi_{R 2 \alpha}(-p+k) \sigma_{\mu}^{-\alpha, \beta} \Psi_{L 1 \beta}(p) \\
& \left.+Q \Psi_{R 0 \alpha}(p-k) \sigma_{\mu}^{-\alpha, \beta} \Psi_{L 0 \beta}(p)\right], \\
& O_{S C^{\prime}, \mu}\left(k \approx k_{F_{R 1}}+k_{F_{L 1}}\right) \\
& =\frac{1}{\sqrt{L}} \sum_{p, \alpha, \beta} \alpha\left[\Psi_{R 1 \alpha}(-p+k) \sigma_{\mu}^{-\alpha, \beta} \Psi_{L 1 \beta}(p)\right], \\
& O_{S C^{\prime \prime}, \mu}\left(k \approx k_{F_{R 1}}+k_{F_{L 0}}\right) \\
& =\frac{1}{\sqrt{L}} \sum_{p, \alpha, \beta} \alpha\left[\Psi_{L 0 \alpha}(-p+k) \sigma_{\mu}^{-\alpha, \beta} \Psi_{R 1 \beta}(p)\right],
\end{aligned}
$$

where for density wave (DW) operators $\mu=0$ stands for CDW and $\mu=1,2,3$ for SDW; while for superconducting (SC) operators $\mu=0$ stands for SS and $\mu=1,2,3$ for TS; $\sigma_{\mu}^{\alpha, \beta}$ are the Pauli matrices, with $\sigma_{0}^{\alpha, \beta}=\mathbf{1}_{2 \times 2}$.

The response functions $\chi$ defined in Eq. (B1) do not obey scaling equations, ${ }^{23}$ but the auxiliary functions defined as $\bar{\chi}(k, l)=\pi v_{F}(d / d l) \chi(k, l)$ do. Finally, the scaling equations for the $\bar{\chi}$ functions read

$$
\begin{gathered}
\frac{\partial}{\partial l} \ln \bar{\chi}_{C D W}=\Delta_{D W}-\frac{2}{\pi v_{F}} g_{1}^{(1)}, \\
\frac{\partial}{\partial l} \ln \bar{\chi}_{S D W}=\Delta_{D W}, \\
\frac{\partial}{\partial l} \ln \bar{\chi}_{C D W^{\prime}}^{P}=\Delta_{D W^{\prime}}-\frac{2}{\pi v_{F}} 2 g_{4}^{(1)}+\frac{P}{\pi v_{F}}\left(g_{1}^{(2)}-2 g_{2}^{(1)}\right), \\
\frac{\partial}{\partial l} \ln \bar{\chi}_{S D W^{\prime}}^{P}=\Delta_{D W^{\prime}}+\frac{P}{\pi v_{F}} g_{1}^{(2)}, \\
\frac{\partial}{\partial l} \ln \bar{\chi}_{C D W^{\prime \prime}}=\Delta_{D W^{\prime \prime}}-\frac{2 \beta}{\pi v_{F}} g^{(1)}, \\
\frac{\partial}{\partial l} \ln \bar{\chi}_{S D W^{\prime \prime}}=\Delta_{D W^{\prime \prime}},
\end{gathered}
$$

$$
\begin{aligned}
\frac{\partial}{\partial l} \ln \bar{\chi}_{C D W^{\prime \prime \prime}}^{P}=\Delta_{D W^{\prime \prime \prime}}-\frac{2 \alpha}{\pi v_{F}} f^{(1)}+\frac{\alpha P}{\pi v_{F}}\left(u_{F}-2 u_{B}\right), & \frac{\partial}{\partial l} \ln \bar{\chi}_{S D W^{\prime \prime \prime}}^{P}=\Delta_{D W^{\prime \prime \prime}}+\frac{\alpha P}{\pi v_{F}} u_{F}, \\
\frac{\partial}{\partial l} \ln \bar{\chi}_{S S}^{P, Q}= & \frac{2}{3} \Delta_{S C}^{(a)}+\frac{1}{3} \Delta_{S C}^{(b)}+\frac{2}{3 \pi v_{F}}\left[-g_{1}^{(1)}-P\left(g_{1}^{(2)}+g_{2}^{(1)}\right)\right] \\
- & \frac{\beta}{3 \pi v_{F}}\left[2(Q+P Q)\left(u_{F}+u_{B}\right)+g^{(1)}\right], \\
\frac{\partial}{\partial l} \ln \bar{\chi}_{T S}^{P, Q}= & \frac{2}{3} \Delta_{S C}^{(a)}+\frac{1}{3} \Delta_{S C}^{(b)}+\frac{2}{3 \pi v_{F}}\left[g_{1}^{(1)}-P\left(g_{1}^{(2)}-g_{2}^{(1)}\right)\right] \\
& -\frac{\beta}{3 \pi v_{F}}\left[2(Q+P Q)\left(u_{F}-u_{B}\right)-g^{(1)}\right], \\
& \frac{\partial}{\partial l} \ln \bar{\chi}_{S S^{\prime}}=\Delta_{S C^{\prime}}-\frac{1}{\pi v_{F}} g_{4}^{(1)}, \\
& \frac{\partial}{\partial l} \ln \bar{\chi}_{T S^{\prime}}=\Delta_{S C^{\prime}}+\frac{1}{\pi v_{F}} g_{4}^{(1)}, \\
& \frac{\partial}{\partial l} \ln \bar{\chi}_{S S^{\prime \prime}}=\Delta_{S C^{\prime \prime}}-\frac{\alpha}{\pi v_{F}} f^{(1)}, \\
& \frac{\partial}{\partial l} \bar{\chi}_{T S^{\prime \prime}}=\Delta_{S C^{\prime \prime}}+\frac{\alpha}{\pi v_{F}} f^{(1)},
\end{aligned}
$$

where the nonperturbative contributions encoded in the anomalous dimensions $\Delta_{D W}, \Delta_{D W^{\prime}}, \Delta_{D W^{\prime \prime}}, \Delta_{D W^{\prime \prime \prime}}, \Delta_{S C}^{(a, b)}, \Delta_{S C^{\prime}}$, $\Delta_{S C^{\prime \prime}}$ are evaluated in Appendix A.

\section{APPENDIX C: INTERTUBE SCREENING FOR FORWARD SCATTERING PROCESSES}

In this appendix we study the Coulomb interaction at small momentum transfer between electrons belonging to different nanotubes in a 3D array. The long-range intertube effects operate in two ways: (i) they provide a screening of the bare intratube forward-scattering couplings $g_{2}^{(2)}, g_{2}^{(4)}, g_{4}^{(2)}, g_{4}^{(4)}, g^{(2)}, g^{(4)}, f^{(2)}, f^{(4)}$, and (ii) they produce new intertube couplings $\widetilde{g}_{2}^{(2)}, \widetilde{g}_{4}^{(2)}$ [see Eqs. (45) and (46)] with nontrivial scaling flow. Here, we take into account the screening effects by the nanotube environment by generalizing the approach devised in Ref. 39, which essentially consists of a RPA treatment of the dielectric constant. The RPA approximation is justified as long as the electrons are not allowed to tunnel between different nanotubes, and we assume that this condition applies to the system under consideration in the main body of the paper. It is important to stress that the screened interactions obtained by this approach do not contain any dependence on the energy cutoff. As a consequence, the RPA screening only imposes the initial value of the forward-scattering couplings in Sec. IV, but does not affect their scaling flow. 
Let $\mathbf{l}$ and $\mathbf{l}^{\prime}$ be the positions of two nanotubes measured over a section of the 3D array, orthogonal to the tube axes. We introduce the long-range bare Coulomb interaction with momentum transfer $k$ along the longitudinal direction by partially Fourier transforming the 3D Coulomb potential

$$
V_{\mathbf{l}, \mathbf{I}^{\prime}}(k)=\frac{2 e^{2}}{\kappa} K_{0}\left(k\left|\mathbf{I}-\mathbf{l}^{\prime}\right|\right),
$$

where $K_{0}(x)$ is the modified Bessel function, which diverges logarithmically as $x \rightarrow 0$ and is exponentially suppressed for $x>1$. It is worth observing that $V_{\mathrm{I}, \mathrm{I}^{\prime}}(k)$ does not take into account the variation of the electronic Bloch wave functions around the waist of the nanotubes. This approximation is justified as long as the transverse momentum transfer is small, i.e., for forward-scattering processes. When $V_{\mathbf{l}, \mathbf{I}^{\prime}}(k)$ is evaluated within the same nanotube, it is understood that $\left|\mathbf{I}-\mathbf{l}^{\prime}\right| \sim R$, and we recover the intratube interaction of $\mathrm{Eq}$. (2), with the logarithimc divergence at small momentum transfer.

The RPA-screened $V_{\mathbf{1}, \mathbf{I}^{\prime}}^{(r)}(k)$ obeys the Dyson equation

$$
V_{\mathbf{1}, \mathbf{I}^{\prime}}^{(r)}(k)=V_{\mathbf{l}, \mathbf{I}^{\prime}}(k)+\Pi \sum_{\mathbf{l}^{\prime \prime}} V_{\mathbf{l}, \mathbf{I}^{\prime \prime}}(k) V_{\mathbf{l}^{\prime \prime}, \mathbf{I}^{\prime}}^{(r)}(k),
$$

where $\mathbf{I}^{\prime \prime}$ runs over all the positions of the nanotubes in the $3 \mathrm{D}$ array and $\Pi$ is the polarizability of the $1 \mathrm{D}$ electron gas; its zero-frequency Fourier transform reads

$$
\Pi(k)=\frac{2}{L} \sum_{q} \frac{f\left(\varepsilon_{q+k}\right)-f\left(\varepsilon_{q}\right)}{\varepsilon_{q+k}-\varepsilon_{q}},
$$

where $f$ is the Fermi-Dirac distribution and $L$ is the lenght of the nanotubes. In order to solve Eq. (C2), we introduce the 2D Fourier transforms of $V_{\mathbf{l}, \mathrm{I}^{\prime}}(k)$ and $V_{\mathrm{l}, \mathrm{I}^{\prime}}^{(r)}(k)$

$$
V_{\mathbf{l}, \mathbf{I}^{\prime}}(k)=\left(\frac{d}{2 \pi}\right)^{2} \int_{B Z} d^{2} \mathbf{p} \phi(k, \mathbf{p}) e^{i \mathbf{p} \cdot\left(\mathbf{l}-\mathbf{l}^{\prime}\right)},
$$

and the same with $V \rightarrow V^{(r)}$ and $\phi \rightarrow \phi^{(r)}$, where $B Z$ indicates the first Brillouin zone of the nanotube lattice in the crosssection of the array, with lattice constant $d \sim 1 \mathrm{~nm}$. In terms of $\phi(k, \mathbf{p})$ and $\phi^{(r)}(k, \mathbf{p})$, the Dyson equation reduces to

$$
\phi^{(r)}(k, \mathbf{p})=\phi(k, \mathbf{p})+\Pi(k) \phi(k, \mathbf{p}) \phi^{(r)}(k, \mathbf{p}) .
$$

This permits us to calculate the screened forward-scattering interaction with longitudinal momentum transfer $k$ between electrons in tubes at $\mathbf{l}$ and $\mathbf{l}^{\prime}$

$$
V_{\mathbf{l}, \mathbf{I}^{\prime}}^{(r)}(k)=\left(\frac{d}{2 \pi}\right)^{2} \int_{B Z} d^{2} \mathbf{p} \frac{\phi(k, \mathbf{p})}{1-\Pi(k) \phi(k, \mathbf{p})} e^{i \mathbf{p} \cdot\left(\mathbf{l}-\mathbf{I}^{\prime}\right)}
$$

The numerical evaluation of $V_{1, \mathbf{l}^{\prime}}^{(r)}(k)$ shows that such interaction is not negligible only for $\mathbf{l}=\mathbf{l}^{\prime}$ and for nearestneighbor tubes, i.e., for $\left|\mathbf{l}-\mathbf{l}^{\prime}\right|=d$. This calls for the introduction of intertube couplings with $\left|\mathbf{l}-\mathbf{l}^{\prime}\right|=d$. As discussed in Sec. IV, two of them $\left(\widetilde{g}_{2}^{(2)}\right.$ and $\left.\widetilde{g}_{4}^{(2)}\right)$ have nontrivial scaling flow, and affect also the scaling of the intratube couplings.

The logarithmic divergence of $V_{\mathbf{l}, \mathrm{I}^{\prime}}(k)$ at $k=0$ is cured by the RPA screening; nevertheless, the effective interactions are still enhanced at small $k$, but they saturate at a finite value. For the intratube interaction we have found that the saturation value is essentially independent of $\kappa$ and is $V_{1,1}^{(r)}$ $\times(k=0) / v_{F} \approx 0.65$. In the intertube case, the RPA screening is much more efficient and the saturation value for $\left|\mathbf{l}-\mathbf{l}^{\prime}\right|$ $=d$ is $V_{\mathbf{l}, \mathbf{I}^{\prime}}^{(r)}(k=0) / v_{F} \approx 0.002 \kappa$, with an approximately linear dependence on $\kappa$, at least up to $\kappa \sim 5$. As discussed above, these asymptotic values are used as initial conditions for the forward-scattering couplings in the scaling equations in Sec. IV.
${ }^{1}$ R. Saito, M. Fujita, G. Dresselhaus, and M. S. Dresselhaus, Appl. Phys. Lett. 60, 2204 (1992); J. W. Mintmire, B. I. Dunlap, and C. T. White, Phys. Rev. Lett. 68, 631 (1992); N. Hamada, S. Sawada, and A. Oshiyama, ibid. 68, 1579 (1992).

${ }^{2}$ Z. Yao, H. W. Ch. Postma, L. Balents, and C. Dekker, Nature (London) 402, 273 (1999).

${ }^{3}$ M. Bockrath, D. H. Cobden, J. Lu, A. G. Rinzler, R. E. Smalley, L. Balents, and P. L. McEuen, Nature (London) 397, 598 (1999).

${ }^{4}$ A. Yu. Kasumov, R. Deblock, M. Kociak, B. Reulet, H. Bouchiat, I. I. Khodos, Yu. B. Gorbatov, V. T. Volkov, C. Journet, and M. Burghard, Science 284, 1508 (1999).

${ }^{5}$ A. F. Morpurgo, J. Kong, C. M. Marcus, and H. Dai, Science 286, 263 (1999).

${ }^{6}$ J. González, Phys. Rev. Lett. 87, 136401 (2001).

${ }^{7}$ M. Kociak, A. Yu. Kasumov, S. Guéron, B. Reulet, I. I. Khodos, Yu. B. Gorbatov, V. T. Volkov, L. Vaccarini, and H. Bouchiat, Phys. Rev. Lett. 86, 2416 (2001).

${ }^{8}$ A. Kasumov, M. Kociak, M. Ferrier, R. Deblock, S. Guéron, B. Reulet, I. Khodos, O. Stéphan, and H. Bouchiat, Phys. Rev. B
68, 214521 (2003).

9 J. González, Phys. Rev. Lett. 88, 076403 (2002); Phys. Rev. B 67, 014528 (2003).

${ }^{10}$ L. Balents and M. P. A. Fisher, Phys. Rev. B 55, R11973 (1997).

${ }^{11}$ R. Egger and A. O. Gogolin, Phys. Rev. Lett. 79, 5082 (1997); Eur. Phys. J. B 3, 281 (1998).

${ }^{12}$ C. Kane, L. Balents, and M. P. A. Fisher, Phys. Rev. Lett. 79, 5086 (1997).

${ }^{13}$ H. Yoshioka and A. A. Odintsov, Phys. Rev. Lett. 82, 374 (1999); A. A. Odintsov and H. Yoshioka, Phys. Rev. B 59, R10457 (1999).

${ }^{14}$ A. A. Nersesyan and A. M. Tsvelik, Phys. Rev. B 68, 235419 (2003).

${ }^{15}$ L. S. Levitov and A. M. Tsvelik, Phys. Rev. Lett. 90, 016401 (2003).

${ }^{16}$ J. V. Alvarez and J. González, Phys. Rev. Lett. 91, 076401 (2003).

${ }^{17}$ A. De Martino and R. Egger, Phys. Rev. B 70, 014508 (2004).

${ }^{18}$ L. X. Benedict, V. H. Crespi, S. G. Louie, and M. L. Cohen, Phys. Rev. B 52, 14935 (1995). 
${ }^{19}$ A. De Martino and R. Egger, Phys. Rev. B 67, 235418 (2003).

${ }^{20}$ Z. K. Tang, L. Zhang, N. Wang, X. X. Zhang, G. H. Wen, G. D. Li, J. N. Wang, C. T. Chan, and P. Sheng, Science 292, 2462 (2001).

${ }^{21}$ H. J. Liu and C. T. Chan, Phys. Rev. B 66, 115416 (2002).

${ }^{22}$ I. Cabria, J. W. Mintmire, and C. T. White, Phys. Rev. B 67, 121406(R) (2003).

${ }^{23}$ J. Sólyom, Adv. Phys. 28, 201 (1979).

${ }^{24}$ Yu. A. Krotov, D.-H. Lee, and S. G. Louie, Phys. Rev. Lett. 78, 4245 (1997).

${ }^{25}$ R. Egger and H. Grabert, Phys. Rev. Lett. 79, 3463 (1997).

${ }^{26}$ V. J. Emery, in Highly Conducting One-Dimensional Solids, edited by J. T. Devreese, R. P. Evrard, and V. E. Van Doren (Plenum, New York, 1979).

${ }^{27}$ D. Loss and T. Martin, Phys. Rev. B 50, 12160 (1994).

${ }^{28}$ D. Connétable, G.-M. Rignanese, J.-C. Charlier, and X. Blase, Phys. Rev. Lett. 94, 015503 (2005).

${ }^{29}$ R. Barnett, E. Demler, and E. Kaxiras, Phys. Rev. B 71, 035429 (2005).

${ }^{30}$ M. T. Figge, M. Mostovoy, and J. Knoester, Phys. Rev. B 65,
$125416(2002)$.

${ }^{31}$ D. W. Wang, A. J. Millis, and S. Das Sarma, Phys. Rev. B 64, 193307 (2001).

${ }^{32}$ K. Kamide, T. Kimura, M. Nishida, and S. Kurihara, Phys. Rev. B 68, 024506 (2003).

${ }^{33}$ J. González, Phys. Rev. B 72, 073403 (2005).

${ }^{34}$ A. Bachtold, M. de Jonge, K. Grove-Rasmussen, P. L. McEuen, M. Buitelaar, and C. Schönenberger, Phys. Rev. Lett. 87, 166801 (2001).

${ }^{35}$ J. González, Eur. Phys. J. B 36, 317 (2003).

${ }^{36}$ F. Triozon, S. Roche, A. Rubio, and D. Mayou, Phys. Rev. B 69, 121410(R) (2004).

${ }^{37}$ This condition is satisfied with great approximation in the systems considered in the paper, as the couplings $f^{(2)}$ and $f^{(4)}$ have equal values at the beginning of the scaling flow and they are very softly renormalized at low temperatures.

${ }^{38}$ A. Sédéki, L. G. Caron, and C. Bourbonnais, Phys. Rev. B 65, 140515(R) (2002).

${ }^{39}$ P. Hawrylak, G. Eliasson, and J. J. Quinn, Phys. Rev. B 37, 10187 (1988). 